DESY 13-074, HU-EP-13/23

April 2013

\title{
The Standard model as a low-energy effective theory: what is triggering the Higgs mechanism?*
}

\author{
Fred JeGERLEHNER,
}

\author{
Humboldt-Universität zu Berlin, Institut für Physik, \\ Newtonstrasse 15, D-12489 Berlin, Germany \\ Deutsches Elektronen-Synchrotron (DESY), \\ Platanenallee 6, D-15738 Zeuthen, Germany
}

\begin{abstract}
The discovery of the Higgs by ATLAS and CMS at the LHC not only provided the last missing building block of the electroweak Standard Model, the mass of the Higgs has been found to have a very peculiar value about $125 \mathrm{GeV}$, which is such that vacuum stability is extending up to the Planck scale. This may have much deeper drawback than anticipated so far. The impact on the running of the SM gauge, Yukawa and Higgs couplings up to the Planck scale has been discussed in several articles recently. Here we consider the impact on the running masses and we discuss the role of quadratic divergences within the Standard Model. The change of sign of the coefficient of the quadratically divergent terms showing up at about $\mu_{0} \sim 7 \times 10^{16} \mathrm{GeV}$ may be understood as a first order phase transition restoring the symmetric phase, while its large negative values at lower scales triggers the Higgs mechanism, running parameters evolve in such a way that the symmetry is restored two orders of magnitude before the Planck scale. Thus, the electroweak phase transition takes place at the scale $\mu_{0}$ and not at the electroweak scale $v \sim 250 \mathrm{GeV}$. The SM Higgs system and its phase transition could play a key role for the inflation of the early universe. Also baryogenesis has to be reconsidered under the aspect that perturbative arguments surprisingly work up to the Planck scale.
\end{abstract}

PACS numbers: 14.65.Ha,12.15.Lk,12.15.Ff

Keywords: phase transitions, renormalization group equation, electroweak radiative corrections

${ }^{*}$ I dedicate this article to the memory of my longtime friend and colleague, Prof. Dr. Jochem Fleischer, who recently passed away. The one-loop on-shell versus $\overline{\mathrm{MS}}$ matching conditions used in the present work we have worked out together more than 30 years ago. 


\section{Introduction}

Evidence strengthens more and more that the new particle discovered by ATLAS [1] and CMS [2] at the LHC at CERN is the last missing state required by the Standard Model (SM) of particle physics, the Higgs boson. For the first time the complete SM spectrum is known now. With its discovery, the mass of the Higgs boson has been established within a narrow range such that all SM parameters (except for the neutrino ones) for the first time are known with remarkable accuracy. One of the interesting consequences is that now we can answer quite reliably the long standing question where the effective SM parameters evolve when going to highest energies. It may be no accident that the observed Higgs mass turned out to match expectations from considerations of SM Higgs vacuum stability bounds, addressed long ago in Ref. [3], for example, and more recently in Refs. [4 11].

Knowing the Higgs mass allows us to say more about the phase structure of the SM. Commonly, quadratic divergences are considered to bring the SM into trouble: the hierarchy, fine tuning or naturalness problem. If one understands the SM as the renormalizable low-energy effective tail of a system existing at the Planck scale, which exhibits the Planck length as a fundamental cutoff, the relation between bare and renormalized parameters acquires a direct physical meaning. The low-energy expansion in the small parameter $x=E / M_{\text {Planck }}$ suggests that only operators of dimension 4 or less are seen at low energies, which means that the low-energy tail is a local renormalizable effective Lagrangian Quantum Field Theory (QFT). As energies increase at some point the first non-renormalizable effective interactions show up: operators of dimension 5 involving fermion fields and operators of dimension 6 which can be build from bosonic fields. Dimension 5 operators yield typically a $0.1 \%$ effect, a typical accuracy achieved in many particle physics experiments, at what is typical for Grand Unified Theory (GUT) scales, namely $10^{16} \mathrm{GeV}$. Dimension 6 operators would yield an effect of similar size at $\sim 3.6 \times 10^{18} \mathrm{GeV}$. So we can expect local renormalizable QFT structure to apply up to about two orders of magnitude below the Planck scale, because the infinite tower of non-renormalizable operators scaling like $x^{n}$ with $n=1,2,3, \cdots$ are irrelevant, i.e. they scale down with increasing powers of the inverse cutoff. The trouble makers are the relevant operators, those which have positive mass dimension: the mass terms in particular. The latter scale like $\Lambda_{\text {Planck }} / m_{f}$ for fermions and like $\Lambda_{\text {Planck }}^{2} / M_{b}^{2}$ for bosons. As relevant operators they have to be tuned in order not to freeze out by acquiring effective masses scaled up by one or two power in the cutoff for fermions or bosons, respectively. In condensed matter physics one would tune, as a typical relevant parameter, the temperature $T$ to its critical value $T_{c}$, in order to let the system built up long range correlations, known as critical phenomena (see e.g. [12]). In particle physics the role of the reduced temperature $\left(T-T_{c}\right) / T_{c}$ is taken by the renormalized particle mass, which has to go to zero in order to see them in the lowenergy spectrum. What is tuning particle masses in the low-energy effective theory? Symmetries as we know! Chiral symmetry protects the fermion masses, local gauge symmetry protects the gauge boson masses, their non-vanishing being a consequence of spontaneous symmetry breaking. The one exception are scalar masses, which only can be protected by doubling the states by pairing all SM particles, supplemented by an additional Higgs doublet, into a supersymmetric extension or by conformal conspir- 
acy when the entire particle content of the SM or an extension of it is such that the fermionic and bosonic degrees of freedom compensate each other collectively. The well known example is Veltman's "Infrared - Ultraviolet Connection" proposed in Ref. [13, which noted that the coefficient of the quadratic divergences could vanish if the sum of properly weighted Higgs, $W$ and $Z$ boson mass-squares would cancel the top quark mass-square contribution (see below).

One of the key indications that the SM is a low-energy effective theory is the occurrence of local gauge symmetries. In particular, the non-Abelian local symmetries are not symmetries in the common sense, like global symmetries. They rather represent a dynamical principle (like the equivalence principle in gravity) implying a special form of the dynamics. One could call them "quantum symmetries" as they determine a form of quantum interference known as gauge cancellations. The latter are well known from processes like $W$-pair production where three Born level diagrams conspire to produce large cancellations of terms growing badly with energy and as a result yield the tamed observable cross section (see e.g. Ref. [14] and references therein). In fact non-Abelian gauge structure is an automatic consequence of a low-energy expansion: it is the only possible residual interaction structure, which is not suppressed by the cutoff (often misleadingly referred to as "tree unitarity" constraint) [15] 17]. Also anomaly cancellations may be understood as low-energy conspiracies, the otherwise non-renormalizable terms are suppressed by inverse powers of the cutoff. The grouping of the SM fermions into families is a consequence of this. For a more general view on the emergence of the SM see Refs. [18,19]. The general set up for the construction of a long range effective theory is Wilson's Renormalization Group (RG) [20] of integrating out short distance fluctuations while keeping the infrared tail. In the infrared Wilson's RG is identical to the bouna fide QFT-RG as we know it in the SM or elsewhere.

While the RG evolution equations in the symmetric phase of the SM have been known for a long time to two loops, recently also three loop result have been calculated in Refs. [8, 9, 11] in the $\overline{\mathrm{MS}}$ scheme, which is very suitable for investigating the highenergy behavior of the SM, which is expected to be represented by the symmetric phase 1 . The more critical point is the experimental values of the $\overline{\mathrm{MS}}$ parameters at the $Z$ boson mass or at the electroweak scale $v=264.22 \mathrm{GeV}$. Most parameters are known from "low-energy" experiments obtained in the real world in the broken phase of the SM, typically in the on-shell renormalization scheme. The transcription of data from the on-shell to the $\overline{\mathrm{MS}}$ scheme is non-trivial within the SM because of non-decoupling effects in the weak sector of the SM at low energies (see e.g. Ref. 21] for a discussion in our context).

Another, maybe more serious, issue which is very different for the EW sector in comparison to QCD, is the appearance of quadratic divergences. They are absent in QCD where in the chiral limit only logarithmic divergences show up. In the electroweak part of the SM, by the fact that spontaneous breaking of the symmetry does not affect the ultraviolet (UV) properties of the theory, quadratic divergences show up in the renormalization of the mass parameter $m^{2}$ of the scalar potential, which in the

\footnotetext{
${ }^{1}$ Note that in the symmetric phase where all field but the Higgses are massless an S-matrix does not exist and correspondingly an on-shell scheme is not defined. The $\overline{\mathrm{MS}}$ parametrization is then a natural parametrization at hand, most closely connected to the bare parameters.
} 
symmetric phase is given by $V=\frac{m^{2}}{2} \phi^{2}+\frac{\lambda}{24} \phi^{4}$, where $\phi$ denotes the real scalar Higgs filed. The limit $m=0$ in the SM is not protected by any symmetry, the famous naturalness or hierarchy problem (i.e. a non-zero quadratically UV divergent $m^{2}$ term in the Lagrangian is induced by renormalization). Besides $m^{2}$, the $U(1)_{Y}$ and $S U(2)_{L}$ gauge couplings $g^{\prime}$ and $g$, respectively, the Yukawa couplings $y_{f}$ and the Higgs selfcoupling $\lambda$ are logarithmically divergent only and their running is governed by the standard RG equations for dimensionless parameters. This carries over to the broken phase which forms the low-energy structure of the SM. The dimensionful parameter $m^{2}$ transmutes to the Higgs mass $M_{H}^{2}=\frac{1}{3} \lambda v^{2} \hat{=} 2 m^{2}$ and since $\lambda$ satisfies a normal RG equation governed by logarithmic divergences only, all quadratic divergences must by exhibited by the Higgs bare vacuum expectation value $v_{b}$, or, equivalently, in the bare Fermi constant $G_{F b}=1 /\left(\sqrt{2} v_{b}^{2}\right)$. Since all masses are proportional to $v$ all masses are affected by the issue of quadratic divergences. In the broken phase the quadratic divergences show up in the tadpole contributions. Renormalizability guarantees that no other type of UV singularities are induced by renormalization.

If we do not take into consideration supersymmetric extensions of the SM, which is a possible solution of the naturalness problem, an alternative possibility within the framework of the SM could be a "conformal conspiracy" 2 collectively between SM particles: the quadratic divergences can be absent if SM fermion contributions balance against the bosonic ones [13]. Only the heavier states are relevant. At the one loop level the quadratic divergences, which in dimensional regularization (DR) show up as poles 3 at $D=2$, are known to be given by

$$
\delta m_{H}^{2}=\frac{\Lambda^{2}}{16 \pi^{2}} C_{1} ; \quad C_{1}=\frac{6}{v^{2}}\left(M_{H}^{2}+M_{Z}^{2}+2 M_{W}^{2}-4 M_{t}^{2}\right)
$$

modulo small lighter fermion contributions. The condition for the absence of the quadratic divergences $C_{1} \simeq 0$ for the given top quark mass would require a Higgs mass $M_{H} \simeq 314.92 \mathrm{GeV}$ in the one-loop approximation. The two loop corrections have been calculated in Refs. [23, 24] with results which do not agree, unfortunately. We will accept the recent result

$$
\begin{aligned}
C_{2}= & C_{1}-2 \frac{\ln \left(2^{6} / 3^{3}\right)}{16 \pi^{2}}\left[\left(-36 M_{t}^{4}+18 M_{H}^{2} M_{t}^{2}+5 / 2 M_{H}^{4}+14 / 3 M_{Z}^{2} M_{t}^{2}-6 M_{Z}^{2} M_{H}^{2}\right.\right. \\
& \left.-77 M_{Z}^{4}-68 / 3 M_{W}^{2} M_{t}^{2}-12 M_{W}^{2} M_{H}^{2}+154 M_{W}^{2} M_{Z}^{2}-320 M_{W}^{4}\right) / v^{4} \\
& \left.+32 g_{3}^{2} M_{t}^{2} / v^{2}\right]
\end{aligned}
$$

of Ref. [24]. It turns out that the two loop correction is moderate such that also the differences in the results do not matter for our purpose. If we require $C_{2} \simeq 0$ we get

\footnotetext{
${ }^{2}$ As in the theory of critical phenomena, long distance (low-energy) effective theories are systematically constructable by applying Wilson's renormalization group approach, and mass parameters similar to the temperature in condensed matter physics have to be tuned to the critical surface in parameter space [18,22. The idea is that the statistical fluctuations at the Planck scale conspire to select modes which are able to survive as long range correlations (light particles). Natural are conspiracies involving few fields: singlets, doublets, triplets as they actually appear in the SM. Note that GUTs are unnatural in such a low energy effective scenario, where symmetries show up because we don't see the details of the underlying model.

${ }^{3}$ The massive scalar tadpole in $D \sim 2$ is independent of $m$ given by $A_{0}(m) \stackrel{D \sim 2}{=} \frac{1}{D-2} \frac{\mu^{2}}{2 \pi}$ while for $D \sim 4$ we obtain $A_{0}(m)=\frac{\Lambda^{2}}{16 \pi^{2}}$ when regularized with an UV cutoff $\Lambda$.
} 
the solution $M_{H} \simeq 276.42 \mathrm{GeV}$ closer but still far away from its phenomenologically established value. Therefore such a possible scenario is ruled out by data, definitely after the Higgs mass has been determined by ATLAS and CMS.

In this paper I advocate that quadratic divergences actually could play an important role in a different way. In fact the coefficient of the quadratic divergence is scale dependent and exhibits a zero not far below the Planck scale as emphasized recently in Ref. 24]. The corresponding change in sign seems to provide a natural explanation for the electroweak symmetry breaking in the SM.

In the next section we remind the reader about the emergence of a local renormalizable QFT in a low energy expansion from a system exhibiting a physical UV cutoff at the microscopic level. In Section 3 we discuss the matching conditions which determine the $\overline{\mathrm{MS}}$ parameters from their physical on-shell counterparts. We emphasize the failure of "decoupling by hand" prescriptions in the weak sector of the SM. The evolution of the SM running parameters up to the Planck scale is presented in Section 4 for couplings, masses and the Higgs vacuum expectation value (VEV). Section 5 is devoted to a discussion of the scale dependence of the quadratic divergences and the observed first order phase transition, which triggers the Higgs mechanism. The impact of the results on inflation scenarios is briefly addressed in Section 6, with an outlook and speculations in Section 7.

\section{Low energy effective QFT of a cutoff system}

If we say that the SM is a low-energy effective theory we mean that there must exist a more fundamental system exhibiting a physical cutoff, as typical for condensed matter systems. Such a system we expect to reside at the Planck scale, and the SM is expected to be the renormalizable tail at long distances relative to the Planck length. The Planck energy scale being beyond any direct experimental access, so far we only know its long range structure and that the underlying fundamental system must be in the universality class of the SM. Let us be more specific and sketch the construction of a low-energy effective QFT by looking at the cutoff version of the Higgs system only, for simplicity:

$$
\mathcal{L}=\mathcal{L}_{0}+\mathcal{L}_{\text {int }}=\frac{1}{2} \partial^{\mu} \phi(x)\left(1+\square / \Lambda^{2}\right) \partial_{\mu} \phi(x)-\frac{1}{2} m_{0}^{2} \phi(x)^{2}-\frac{\lambda_{0} \Lambda^{\varepsilon}}{4 !} \phi^{4}(x) .
$$

The regularization is chosen here as a Pais-Uhlenbeck higher-derivative kinetic term [25], which is equivalent to a Pauli-Villars cutoff [26]. We are interested in the model for $D=$ 4 space-time dimensions but may consider the more general case in $D=4-\varepsilon$ dimensions with $2 \leq D \leq 4$ in order to make comparisons with the same model in dimensional regularization. It is characterized by its vertex functions (connected amputated one-particle irreducible diagrams) of $N$ scalar fields $\Gamma_{\Lambda, b}^{(N)}\left(p ; m_{0}, \lambda_{0}\right)=\left\langle\tilde{\phi}\left(p_{1}\right) \cdots \tilde{\phi}\left(p_{N-1}\right) \phi(0)\right\rangle^{\text {prop }}$ as a function of the set of independent momenta, which we denote by $p \hat{=}\left\{p_{i}\right\}(i=$ $1, \cdots, N-1$ ). The bare functions are related to the renormalized ones by (for specific renormalization conditions see Ref. [27]) reparametrizing parameters and fields

$$
\Gamma_{\Lambda r}^{(N)}(p ; m, \lambda)=Z^{N / 2}(\Lambda / m, \lambda) \Gamma_{\Lambda b}^{(N)}\left(p ; \Delta m_{0}(\Lambda, m, \lambda), \lambda_{0}(\Lambda / m, \lambda)\right) .
$$


They satisfy a RG equation for the response to a change of the cutoff $\Lambda$ for fixed renormalized parameters $\left.\Lambda \frac{\partial}{\partial \Lambda} \Gamma(N)_{\Lambda b}\right|_{m, \lambda}$, which by applying the chain rule of differentiation reads

$$
\begin{aligned}
& \left(\Lambda \frac{\partial}{\partial \Lambda}+\beta_{0} \frac{\partial}{\partial \lambda}-N \gamma_{0}+\delta_{0} \Delta m_{0}^{2} \frac{\partial}{\partial \Delta m_{0}^{2}}\right) \Gamma_{\Lambda b}^{(N)}\left(p ; m_{0}, \lambda_{0}\right) \\
= & Z^{-N / 2} \Lambda \frac{\partial}{\partial \Lambda} \Gamma_{\Lambda r}^{(N)}(p ; m, \lambda) .
\end{aligned}
$$

$m_{0 c}^{2}$ is the "critical value" of the bare mass for which the renormalized mass is zero, i.e. $\left.\Gamma_{\Lambda b}^{(2)}\right|_{p=0}=0$, and $\Delta m_{0}^{2}=m_{0}^{2}-m_{0 c}^{2}$. Since the renormalized vertex functions have a regular limit as $\Lambda \rightarrow \infty$, to all orders in perturbation theory the inhomogeneous part behaves as

$$
Z^{N / 2} \Lambda \frac{\partial}{\partial \Lambda} \Gamma_{\Lambda r}^{(N)}(p ; m, \lambda)=O\left(\Lambda^{-2}(\ln \Lambda)^{l}\right),
$$

i.e., the inhomogeneous part, representing a cutoff insertion, falls off faster than the 1.h.s. of Eq. (5) by two powers in the cutoff for large cutoffs. This is easy to understand given the fact that the cutoff enters $\mathcal{L}$ as a term proportional to $\Lambda^{-2}$. Beyond perturbation theory one would have to require the condition

$$
Z^{N / 2} \Lambda \frac{\partial}{\partial \Lambda} \Gamma_{\Lambda r}^{(N)}(p ; m, \lambda) / \Gamma_{\Lambda b}^{(N)}=O\left(\Lambda^{-\eta}\right)
$$

for some positive $\eta$. In addition, also all the RG equation coefficients exist as non-trivial functions in the limit of infinite cutoff:

$$
\lim _{\Lambda \rightarrow \infty} \alpha_{0}(\Lambda / m, \lambda)=\alpha(\lambda), \alpha=\beta, \gamma, \delta
$$

for dimensions $2 \geq D \geq 4$. In $D=4-\varepsilon$ dimensions the proper vertex-functions have a large cutoff $\Lambda$-expansion (see Ref. [28])

$$
\Gamma_{\Lambda b}^{(N)}\left(p ; \Delta m_{0}, \lambda_{0}\right)=\sum_{j, k, l \geq 0} \Lambda^{-2 j-\varepsilon k}(\ln \Lambda)^{l} f_{j k l}^{(N)}\left(p \Delta m_{0}, \lambda_{0} \Lambda^{\varepsilon}\right)
$$

and for large $\Lambda$ we obtain the preasymptote of $\Gamma_{\Lambda b}^{(N)}$

$$
\Gamma_{\Lambda \text { as }}^{(N)}\left(p ; \Delta m_{0}, \lambda_{0}\right)=\sum_{k, l \geq 0} \Lambda^{-\varepsilon k}(\ln \Lambda)^{l} f_{0 k l}^{(N)}\left(p \Delta m_{0}, \lambda_{0} \Lambda^{\varepsilon}\right)
$$

such that

$$
\left|\Gamma_{\Lambda b}^{(N)}\left(p ; \Delta m_{0}, \lambda_{0}\right)-\Gamma_{\Lambda \text { as }}^{(N)}\left(p ; \Delta m_{0}, \lambda_{0}\right)\right|=O\left(\Lambda^{-2}\left(\ln \Lambda^{l_{x}}\right)\right)
$$

where $l_{x}$ is bounded to all orders in the perturbation expansion. The key point is that the still cutoff dependent preasymptote satisfies a homogeneous RG equation, a special property of the long range tail of the bare theory:

$$
\begin{aligned}
& \left(\Lambda \frac{\partial}{\partial \Lambda}+\beta_{\mathrm{as}}\left(\Lambda / \Delta m_{0}, \lambda_{0}\right) \frac{\partial}{\partial \lambda_{0}}-N \gamma_{\mathrm{as}}\left(\Lambda / \Delta m_{0}, \lambda_{0}\right)\right. \\
& \left.\quad+\delta_{\mathrm{as}}\left(\Lambda / \Delta m_{0}, \lambda_{0}\right) \Delta m_{0}^{2} \frac{\partial}{\partial \Delta m_{0}^{2}}\right) \Gamma_{\Lambda \text { as }}^{(N)}\left(p ; \Delta m_{0}, \lambda_{0}\right)=0 .
\end{aligned}
$$


For more details see Refs. [27 29]. The homogeneity tells us that $\Lambda$ has lost it function as a cutoff and takes the role of a renormalization scale, i.e., Eq. (12) represents the response of a rescaling of the system: a change in $\Lambda$ is compensated by a finite renormalization of the fields, the couplings and the massest 4 . By a finite renormalization we may reparametrize the preasymptote by imposing appropriate renormalization conditions. Then there exist a rescaling $\Lambda=\kappa \mu$ such that we obtain the usual RG in the renormalization scale $\mu$ of a non-trivial continuum QFT. This allows for a precise interrelation to $\overline{\mathrm{MS}}$ renormalized quantities. Thus, what we observe as the SM is a physical reparametrization (renormalization) of the preasymptotic bare theory. In the language of critical phenomena the "bare world" at the Planck scale has to be in the universality class of the SM. As we only observe the tail, details of the bare world remain largely unknown. One of the impacts of the very high Planck scale is that the local renormalizable QFT structure of the SM is presumably valid up to what is a typical GUT scale. It has nothing to do with grand unification though. This also justifies the application of the SM RG up to high scales. The tuning "to criticality" of the bare mass to the critical mass $m_{0 c}$ correspond to what is known as the hierarchy or naturalness problem in the SM. This naturalness problem is asking for an answer to the question "who is tuning the knob of the thermostat to adjust the temperature to its critical value (which is determined by the underlying atomic structure of the condensed matter system)". In the symmetric phase of the SM, where there is only one mass (the others are forbidden by the known chiral and gauge symmetries), which is the same for all fields in the Higgs doublet, the fine tuning has the form

$$
m_{0}^{2}=m^{2}+\delta m^{2} ; \delta m^{2}=\frac{\Lambda^{2}}{16 \pi^{2}} C
$$

with a coefficient typically $C=O(1)$. To keep the renormalized mass at some small value, which can be seen at low energy, $m_{0}^{2}$ has to be adjusted to compensate the huge number $\delta m^{2}$ such that about 35 digits must be adjusted in order to get the observed value around the electroweak scale. This is a problem only for a scenario which takes the relation between bare and renormalized theory serious, like in a condensed matter system. The difference is of course that in particle physics we never will be able to directly access experimentally the bare system sitting at the Planck scale. In the

${ }^{4}$ The response of the on-shell renormalized theory to a change in the mass, in the limit $\Lambda \rightarrow \infty$, is given by the Callan-Symanzik equation (see Refs. [30,31])

$$
\left(m \frac{\partial}{\partial m}+\beta(\lambda) \frac{\partial}{\partial \lambda}-N \gamma(\lambda)\right) \Gamma_{r}^{(N)}(p ; m, \lambda)=-m^{2}(2-\delta(\lambda)) \Delta_{0} \Gamma_{r}^{(N)}(p ; m, \lambda),
$$

where $\Delta_{0}$ is the integrated mass operator insertion. For large momenta the r.h.s. is suppressed $O\left(m^{2} \ln (m)^{l}\right)$ by the small mass-square $m^{2} \ll p^{2}$ up to logarithms, such that for large momenta asymptotically

$$
\left(m \frac{\partial}{\partial m}+\beta(\lambda) \frac{\partial}{\partial \lambda}-N \gamma(\lambda)\right) \Gamma_{r a s}^{(N)}(p ; m, \lambda)=0 .
$$

The mass asymptotically only plays the role of a renormalization scale, $\Gamma_{r a s}^{(N)}(p ; m, \lambda)$ are vertex functions of an effectively massless theory. Up to appropriate finite reparametrization and a rescaling $m=\kappa \mu$ the homogeneous CS equations are nothing but the standard RG equation in the $\overline{\mathrm{MS}}$ scheme. 
following we consider the SM as a strictly renormalizable theory, regularized as usual by dimensional regularization 32 in $D=4-\varepsilon$ space-time dimensions, such that the $\overline{\mathrm{MS}}$ parametrization and the corresponding RG can be used in the well known form [33].

\section{Matching conditions}

When studying the scale dependence of a theory at very high energies, where the theory is effectively massless and hence practically in the symmetric phase, the $\overline{\mathrm{MS}}$ renormalization scheme is the favorite choice to study the scale dependence of the theory. On the other hand the physical values of parameters are determined by physical processes described by on-shell matrix elements and thus usually are available in the on-shell renormalization scheme primarily. The transition from one scheme to the other is defined by appropriate matching conditions. For the physical masses they are given by the mass counterterms relating the bare and the renormalized masses as $m_{b 0}^{2}=M_{b}^{2}+$ $\delta M_{b}^{2}$ for bosons and $m_{f 0}=M_{f}+\delta M_{f}$ for fermions, respectively. By $m_{i 0}$ we denoted the bare, by $m_{i}$ the $\overline{\mathrm{MS}}$ and by $M_{i}$ the on-shell masses. Reg $=\frac{2}{\varepsilon}-\gamma+\ln 4 \pi+\ln \mu_{0}^{2}$ is the UV regulator term to be set equal to $\ln \mu^{2}$ where $\mu_{0}$ is the bare $\mu$-parameter and $\mu$ the renormalized one. The substitution defines the UV finite $\overline{\mathrm{MS}}$ parametrization. By identifying $m_{b}^{2}=M_{b}^{2}+\left.\delta M_{b}^{2}\right|_{\operatorname{Reg}=\ln \mu^{2}}$ and $m_{f}=M_{f}+\left.\delta M_{f}\right|_{\operatorname{Reg}=\ln \mu^{2}}$, respectively, we then obtain the $\overline{\mathrm{MS}}$ masses in terms of the on-shell masses. More precisely, this follows from the following relations valid for bosons:

$$
m_{b 0}^{2}=M_{b}^{2}+\left.\delta M_{b}^{2}\right|_{\mathrm{OS}}=m_{b}^{2}+\left.\delta M_{b}^{2}\right|_{\overline{\mathrm{MS}}}
$$

where

$$
\left.\delta M_{b}^{2}\right|_{\overline{\mathrm{MS}}}=\left(\left.\delta M_{b}^{2}\right|_{\mathrm{OS}}\right)_{\mathrm{UV} \text { sing }}
$$

which means that only the ultraviolet (UV) singular Reg terms are kept as $\overline{\mathrm{MS}}$ counterterms. Thus

$$
m_{b}^{2}=M_{b}^{2}+\left.\delta M_{b}^{2}\right|_{\mathrm{OS}}-\left.\delta M_{b}^{2}\right|_{\overline{\mathrm{MS}}}=M_{b}^{2}+\left(\left.\delta M_{b}^{2}\right|_{\mathrm{OS}}\right)_{\mathrm{Reg}=\ln \mu^{2}} .
$$

Corresponding linear relations hold for the fermion masses. Similar relations apply for the coupling constants $g, g^{\prime}, \lambda$ and $y_{f}$, which, however, usually are fixed using the mass-coupling relations in terms of the masses and the Higgs VEV, which is determined by the Fermi constant $G_{F}=G_{\mu}$ as $v=\left(\sqrt{2} G_{\mu}\right)^{-1 / 2}$.

In the mass relations just presented, tadpole contributions have to be included in order to get a gauge invariant relationship between on-shell and $\overline{\mathrm{MS}}$ masses as well as in order to preserve the UV singularity structure and hence the RG equations. Tadpoles show up as renormalization counterterms of the Higgs VEV $v$ and quantities which depend on it, in particular the masses, which are generated by the Higgs mechanism.

The proper expressions including the relevant tadpole terms for the SM counterterms at one-loop have been given in [34] and may be found in the Appendix. For the Higgs mass such a relation has been elaborated in [35] as a relation between $\lambda$ and $\lambda_{\overline{\mathrm{MS}}}$ under the proviso that $G_{F}^{\overline{\mathrm{MS}}}=G_{\mu}$, which is not generally true, because in general $G_{F}^{\overline{\mathrm{MS}}}$ is expected to be a running parameter as well. Interpreted as a relation 
between $m_{H}$ and $M_{H}$, the relation is identical to what is obtained from the relation $m_{H}^{2}=M_{H}^{2}+\left(\left.\delta M_{H}^{2}\right|_{\mathrm{OS}}\right)_{\mathrm{Reg}=\ln \mu^{2}}$. Note that the only information we have on $\lambda$ is from the experimental results on $M_{H}$ via $\lambda=3 \sqrt{2} G_{\mu} M_{H}^{2}$. For the top quark mass the full $\mathrm{SM}$ relation between the pole mass and the $\overline{\mathrm{MS}}$ mass has been evaluated recently in Ref. [21], evaluating known results from Refs. [36-42] (see also [43,44] and comments in Ref. [21]) in the relation

$$
M_{t}-m_{t}\left(\mu^{2}\right)=m_{t}\left(\mu^{2}\right) \sum_{j=1}\left(\frac{\alpha_{s}\left(\mu^{2}\right)}{\pi}\right)^{j} \rho_{j}+m_{t}\left(\mu^{2}\right) \sum_{i=1 ; j=0}\left(\frac{\alpha\left(\mu^{2}\right)}{\pi}\right)^{i}\left(\frac{\alpha_{s}\left(\mu^{2}\right)}{\pi}\right)^{j} r_{i j} \cdot
$$

There is an almost perfect cancellation between the QCD and EW effects for the now known value of the Higgs boson mass. While $\left[m_{t}\left(M_{t}^{2}\right)-M_{t}\right]_{\mathrm{QCD}}=-10.38 \mathrm{GeV}$ one finds [21] $\left[m_{t}\left(M_{t}^{2}\right)-M_{t}\right]_{\mathrm{SM}}=1.14 \mathrm{GeV}$ for $M_{H}=125 \mathrm{GeV}$.

As elaborated in Ref. [21] some care is required in the evaluation of the matching conditions. It is important the remind that the Appelquist-Carazzone theorem [45] does not apply to the weak sector of the SM, i.e. we cannot parametrize and match together effective theories by switching off fields of mass $M>\mu$ at a given scale $\mu$. As we know the theorem applies to QCD and QED, and in these cases provides the basis for the "decoupling by hand" prescription usually used in conjunction with the $\overline{\mathrm{MS}}$ parametrization, the preferred parametrization in perturbative QCD. The nondecoupling in the weak sector of the SM is a consequence of the mass coupling relations, which follow if the masses are generated by the Higgs mechanism. An important question then is what role tadpoles play in implementing the matching conditions, since tadpoles potentially give large contributions. However, we may take advantage of the fact that tadpole contributions drop out from relations between physical (on-shell) parameters and amplitudes [46], while they can produce large shifts in the relations between the "quasi-bare" $\overline{\mathrm{MS}}$ parameters and the on-shell ones. As mentioned before, potentially, the Higgs VEV $v$, which determines the Fermi constant via $G_{F}=\frac{1}{\sqrt{2} v^{2}}$, could be particularly affected. However, comparing the low energy effective Fermi constant $G_{F}$ given by $G_{\mu}$, which is determined by the muon lifetime observed in $\mu$-decay, with its "high energy" variant at the $W$ boson mass scale, where it can be identified with $\hat{G}_{\mu}=\frac{12 \pi \Gamma_{W \ell_{\nu}}}{\sqrt{2} M_{W}^{3}}$ in terms of the leptonic $W$ decay rate. The fact that $\hat{G}_{\mu} \approx G_{\mu}$ with good accuracy is not surprising because the tadpole corrections which potentially lead to substantial corrections are absent in relations between observable quantities as we know. Therefore, a SM parametrization in terms of $\alpha\left(M_{Z}\right), \alpha_{s}\left(M_{Z}\right), G_{\mu}$ and $M_{Z}$ (besides the other masses), provides a good parametrization of the observables extracted from experiments at the vector boson mass scale. While $\alpha\left(M_{Z}\right)$ and $\alpha_{s}\left(M_{Z}\right)$ are strongly scale dependent, $G_{\mu}$ remains unrenormalized below the $Z$ mass scale, which thus is an ideal matching scale, to evaluate the $\overline{\mathrm{MS}}$ parameters in terms of corresponding on-shell values. Usually the scale independence of the effective $G_{F}$ below the $W$ mass scale is "explained" by a "decoupling by hand" argument by inspection of the one-loop RG equation

$$
\mu^{2} \frac{d \ln G_{F}^{\overline{\mathrm{MS}}}}{d \mu^{2}}=\frac{\sqrt{2} G_{F}^{\overline{\mathrm{MS}}}}{8 \pi^{2}}\left\{\sum_{f}\left(m_{f}^{2}-4 \frac{m_{f}^{4}}{m_{H}^{2}}\right)-3 m_{W}^{2}+6 \frac{m_{W}^{4}}{m_{H}^{2}}-3 / 2 m_{Z}^{2}+3 \frac{m_{Z}^{4}}{m_{H}^{2}}+3 / 2 m_{H}^{2}\right\},
$$


which follows from the counterterm given first in Ref. [34]. If we sum terms only over $m<\mu$, there is effectively no running, because of the smallness of the light fermion masses. $G_{F}$ is expected to start running once $M_{W}, M_{Z}, M_{H}$ and $M_{t}$ come into play. At higher scales, certainly, the $\overline{\mathrm{MS}}$ version of $v\left(\mu^{2}\right)$ or equivalently $G_{F}^{\overline{\mathrm{MS}}}\left(\mu^{2}\right)$ must be running as required by the corresponding $\mathrm{RG}$.

For numerical results presented in some of the following sections we use values for the input parameters from Ref. [47]:

$$
\begin{aligned}
& M_{Z}=91.1876(21) \mathrm{GeV}, \quad M_{W}=80.385(15) \mathrm{GeV}, \quad M_{t}=173.5(1.0) \mathrm{GeV}, 5 \\
& G_{\mu}=1.16637 \times 10^{-5} \mathrm{GeV}^{-2}, \quad \alpha^{-1}=137.035999, \quad \alpha_{s}\left(M_{Z}^{2}\right)=0.1184(7) .
\end{aligned}
$$

For the Higgs mass we adopt

$$
M_{H}=125.5 \pm 1.5 \mathrm{GeV},
$$

in accord with latest ATLAS and CMS reports. Furthermore, we take the effective fine-structure constant at the $Z$ boson mass scale to be $\alpha^{-1}\left(M_{Z}^{2}\right)=127.944$. All lightfermion masses $M_{f}(f \neq t)$ give negligible effects and do not play any role in our consideration. The top quark mass given above is taken to be the pole mass.

\section{The SM RG evolution to the Planck scale}

The SM RG in the symmetric phase to two loops has been known for a long time [48. More recently important extensions to three loops have been presented in Refs. [5-11, 24]. Especially the behavior of the Higgs self-coupling $\lambda$ plays a key role for the possible stability or instability of the SM ground state. In fact solutions depend crucially on including all couplings contributing. For example, it makes a big difference whether one works in the gaugeless limit 6 in the evolution of $y_{t}$ and $\lambda$ as in Ref. [9] or is including also the gauge coupling contributions as far as they are known (see e.g. Ref. [10] for a fairly complete set of known corrections). Some time ago RG equations to two loops for the SM masses as well as for the Higgs VEV in the broken phase have been calculated in Ref. 49] where it has been shown that the RG equations of the symmetric phase are correctly obtained from the ones in the broken phase. The inclusion of the tadpoles thereby is crucial.

The RG equation for $v^{2}\left(\mu^{2}\right)$ follows from the RG equations for masses and massless coupling constants using one of the relations

$$
v^{2}\left(\mu^{2}\right)=4 \frac{m_{W}^{2}\left(\mu^{2}\right)}{g^{2}\left(\mu^{2}\right)}=4 \frac{m_{Z}^{2}\left(\mu^{2}\right)-m_{W}^{2}\left(\mu^{2}\right)}{g^{\prime 2}\left(\mu^{2}\right)}=2 \frac{m_{f}^{2}\left(\mu^{2}\right)}{y_{f}^{2}\left(\mu^{2}\right)}=3 \frac{m_{H}^{2}\left(\mu^{2}\right)}{\lambda\left(\mu^{2}\right)} .
$$

As a key relation we will use [49]

$$
\mu^{2} \frac{d}{d \mu^{2}} v^{2}\left(\mu^{2}\right)=3 \mu^{2} \frac{d}{d \mu^{2}}\left[\frac{m_{H}^{2}\left(\mu^{2}\right)}{\lambda\left(\mu^{2}\right)}\right] \equiv v^{2}\left(\mu^{2}\right)\left[\gamma_{m^{2}}-\frac{\beta_{\lambda}}{\lambda}\right]
$$

\footnotetext{
${ }^{6}$ We use this term for the approximation $g^{\prime}=g=0$. The QCD coupling $g_{3}$ in any case has to be taken into account, besides the top Yukawa coupling $y_{t}$ and the Higgs self-coupling $\lambda$.
} 
We remind that all dimensionless couplings satisfy the same RG equations in the broken and in the unbroken phase.

As we know the Higgs VEV $v$ is a key parameter of the SM which interrelates masses and couplings in a well defined way. As a consequence the RG for mass parameters can be obtained not only by direct calculation in the broken phase, but also from the knowledge of the RG of the parameters in the symmetric phase together with the one for $v\left(\mu^{2}\right)$ or $v^{2}\left(\mu^{2}\right)=1 /\left(\sqrt{2} G_{F}^{\overline{\mathrm{MS}}}\left(\mu^{2}\right)\right)$ as given in Eq. (24). The proper $\overline{\mathrm{MS}}$ definition of a running fermion mass is

$$
m_{f}\left(\mu^{2}\right)=\frac{1}{\sqrt{2}} v\left(\mu^{2}\right) y_{f}\left(\mu^{2}\right) .
$$

Of particular interest in our context is the top quark mass for which the RG equation reads

$$
\mu^{2} \frac{d}{d \mu^{2}} \ln m_{t}^{2}=\gamma_{t}\left(\alpha_{s}, \alpha\right)
$$

We split $\gamma_{t}\left(\alpha_{s}, \alpha\right)$ into two parts $\gamma_{t}\left(\alpha_{s}, \alpha\right)=\gamma_{t}^{Q C D}+\gamma_{t}^{E W}$, where $\gamma_{t}^{Q C D}$ is the QCD anomalous dimension, and $\gamma_{t}^{E W}$ the corresponding electroweak one. $\gamma_{t}^{Q C D}$ includes all terms which are proportional to powers of $\alpha_{s}$ only and $\gamma_{t}^{E W}$ includes all other terms proportional to at least one power of $\alpha$, and beyond one-loop multiplied by further powers of $\alpha$ and/or $\alpha_{s}$.

In Ref. [49] the electroweak contribution to the fermion mass anomalous dimension $\gamma_{f}^{E W}$ has been calculated in terms of the RG functions of the parameters in the unbroken phase of the SM: the result is given by

$$
\gamma_{t}^{E W}=\gamma_{y_{t}}+\frac{1}{2} \gamma_{m^{2}}-\frac{1}{2} \frac{\beta_{\lambda}}{\lambda}
$$

where $\gamma_{m^{2}} \equiv \mu^{2} \frac{d}{d \mu^{2}} \ln m^{2}, \beta_{\lambda} \equiv \mu^{2} \frac{d}{d \mu^{2}} \lambda$, and $\gamma_{y_{q}} \equiv \mu^{2} \frac{d}{d \mu^{2}} \ln y_{q}$, with $y_{q}$ the quark Yukawa coupling.

In the following we present the results for the running SM parameters in various plots. The RG equations for the gauge couplings $g_{3}=\left(4 \pi \alpha_{s}\right)^{1 / 2}, g_{2}=g$ and $g_{1}=g^{\prime}$, for the Yukawa coupling $y_{t}$ and for the Higgs potential parameters $\lambda$ and $\ln m^{2}$ have been solved in the $\overline{\mathrm{MS}}$ scheme with initial values obtained by evaluating the matching conditions between pole and running masses. For the case of the dimensionless couplings we reproduce known results within uncertainties. The $\overline{\mathrm{MS}}$ Higgs VEV square is then obtained by $v^{2}\left(\mu^{2}\right)=\frac{6 m^{2}\left(\mu^{2}\right)}{\lambda\left(\mu^{2}\right)}$ and the other masses by the relations (23).

Fig. 1 shows the solutions of the RG equations and the $\beta$-functions up to $\mu=$ $M_{\text {Planck }}$. The running masses and the solutions for the Higgs potential mass parameter $m$ as well as $v$ and the equivalent $G_{F}$ are depicted in Fig. 2

Remarkably, as previously found for the running couplings in Refs. [5, 6, 10, 24, all parameters stay in bounded ranges up to the Planck scale if one adopts our matching conditions together with the so far calculated RG coefficients. We note that including all known terms no transition to a metastable state in the effective Higgs potential is observed, i.e. no change of sign in $\lambda$ occurs, in agreement with Refs. [5, 10]. 

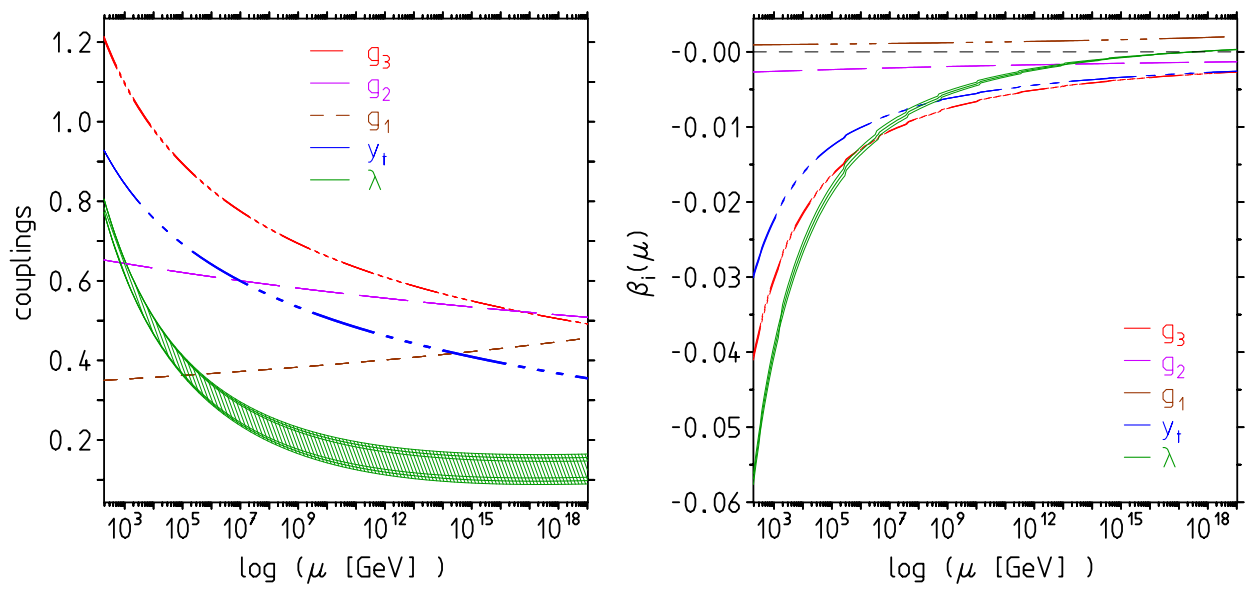

Figure 1: Left: the SM dimensionless couplings in the $\overline{\mathrm{MS}}$ scheme as a function of the renormalization scale (see [5, 6, 10, 24]). The input parameter uncertainties as given in Eqs. (21) and (22) are exhibited by the line thickness. The green band corresponds to Higgs masses in the range [124-127] GeV. Right: the $\beta$-functions for the couplings $g_{3}, g_{2}, g_{1}, y_{t}$ and $\lambda$. The uncertainties are represented by the line widths.

We observe that the various couplings evolve to values of similar (within a factor of about 5) magnitude at the Planck scale. While the gauge coupling are much closer than they are at low energies, there is no reason for perfect unification. The different types, the gauge, the top-Yukawa and the Higgs couplings have no reason not to differ even if they emerge form one cutoff system. That they are of the same order of magnitude, however, makes sense in this kind of scenario.

The key point of the behavior we may understand when we look at the leading terms of the $\beta$-functions. At the $Z$ boson mass scale the couplings are given by $g_{1} \simeq 0.350$, $g_{2} \simeq 0.653, g_{3} \simeq 1.220, y_{t} \simeq 0.935$ and $\lambda \simeq 0.796$. While the gauge couplings behave as expected, $g_{1}$ is infrared (IR) free, $g_{2}$ and $g_{3}$ are asymptotically (ultraviolet) free (AF), with leading coefficients exhibiting the related coupling only,

$$
\beta_{1}=\frac{41}{6} g_{1}^{3} c \simeq 0.00185 ; \quad \beta_{2}=-\frac{19}{6} g_{2}^{2} c \simeq-0.00558 ; \quad \beta_{3}=-7 g_{3}^{3} c \simeq-0.08045,
$$

with $c=\frac{1}{16 \pi^{2}}$, the leading top Yukawa $\beta$-function given by

$$
\begin{aligned}
\beta_{y_{t}} & =\left(\frac{9}{2} y_{t}^{3}-\frac{17}{12} g_{1}^{2} y_{t}-\frac{9}{4} g_{2}^{2} y_{t}-8 g_{3}^{2} y_{t}\right) c \\
& \simeq 0.02328-0.00103-0.00568-0.07046 \\
& \simeq-0.05389
\end{aligned}
$$

not only depends on $y_{t}$, but also on mixed terms with the gauge couplings which have a negative sign. In fact the QCD correction is the leading contribution and determines the behavior. Notice the critical balance between the dominant strong and the top Yukawa couplings: QCD dominance requires $g_{3}>\frac{3}{4} y_{t}$ in the gaugeless limit. 

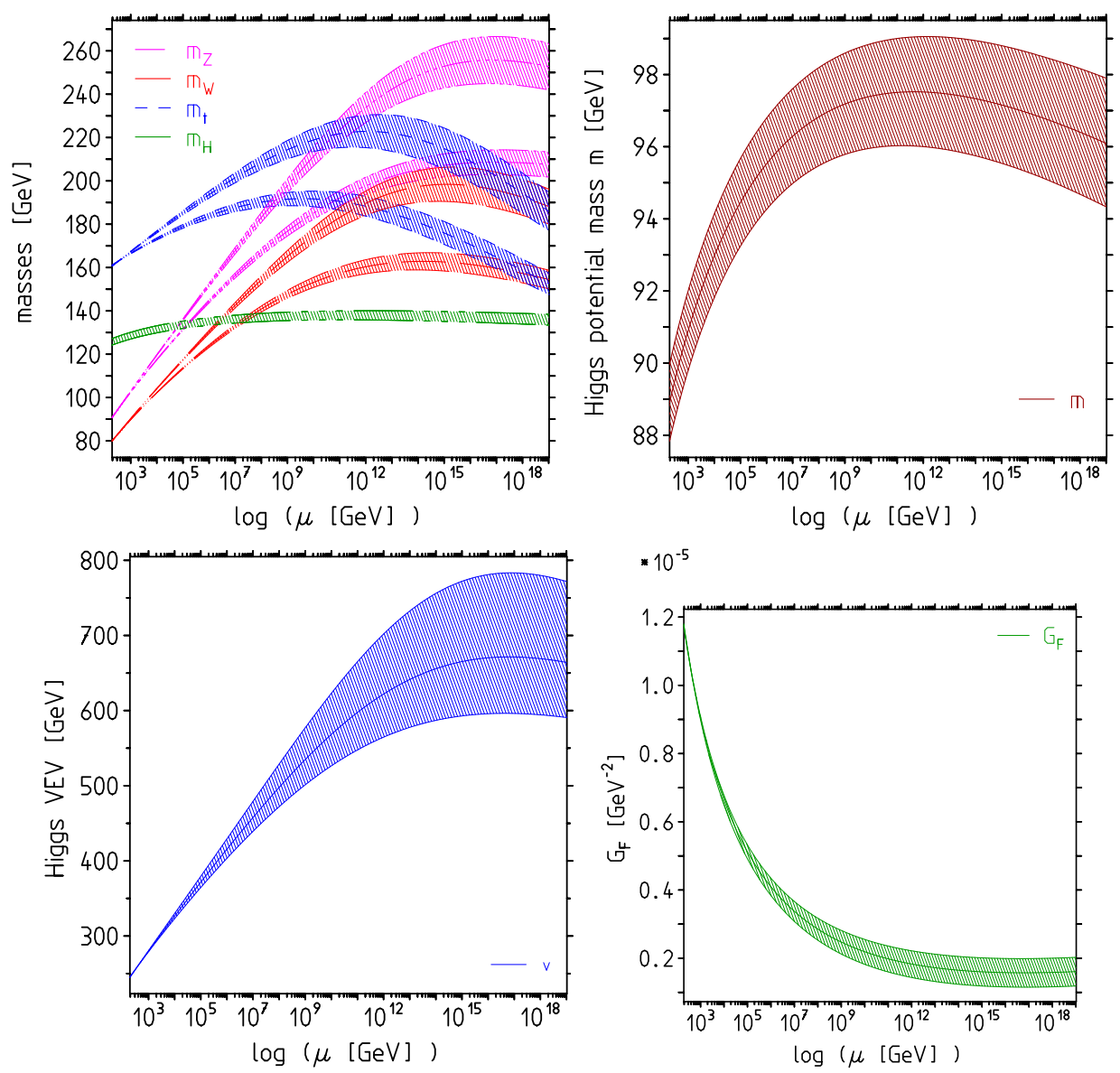

Figure 2: Non-zero dimensional $\overline{\mathrm{MS}}$ running parameters. Top left: the running $\overline{\mathrm{MS}}$ masses. The shadowed regions show parameter uncertainties, mainly due to the uncertainty in $\alpha_{s}$, for a Higgs mass of $124 \mathrm{GeV}$, higher bands, and for $127 \mathrm{GeV}$, lower bands. The range also determines the green band for the Higgs mass evolution. Top right: the $\overline{\mathrm{MS}}$ Higgs potential parameter $m$. Bottom: $v=\sqrt{6 / \lambda} m$ and $G_{F}=1 /\left(\sqrt{2} v^{2}\right)$. Error bands include SM parameter uncertainties and a Higgs mass range $125.5 \pm 1.5 \mathrm{GeV}$ which essentially determines the widths of the bands.

Similarly, the $\beta$-function of the Higgs self-coupling, given by

$$
\begin{aligned}
\beta_{\lambda} & =\left(4 \lambda^{2}-3 g_{1}^{2} \lambda-9 \lambda g_{2}^{2}+12 y_{t}^{2} \lambda+\frac{9}{4} g_{1}^{4}+\frac{9}{2} g_{1}^{2} g_{2}^{2}+\frac{27}{4} g_{2}^{4}-36 y_{t}^{4}\right) c \\
& \simeq 0.01606-0.00185-0.01935+0.05287+0.00021+0.00149+0.00777-0.17407 \\
& \simeq-0.11687
\end{aligned}
$$

is dominated by the top Yukawa contribution and not by the $\lambda$ coupling itself. At leading order it is not subject to QCD corrections. Here, the $y_{t}$ dominance condition reads $\lambda<\frac{3(\sqrt{5}-1)}{2} y_{t}^{2}$ in the gaugeless limit. The top Yukawa coupling is turned from an intrinsically IR free to an AF coupling by the QCD term and similarly the Higgs self-coupling is transmuted from IR free to AF by the dominating top Yukawa term. 
Including known higher order terms, except from $\beta_{\lambda}$, which exhibits a zero at about $\mu_{\lambda} \sim 10^{17} \mathrm{GeV}$, all other $\beta$-functions do not exhibit a zero in the range from $\mu=M_{Z}$ to $\mu=M_{\text {Planck }}$. So apart form the $U(1)_{Y}$ coupling $g_{1}$, which increases only moderately, all other couplings decrease and perturbation theory is in good condition. Actually at $\mu=M_{\text {Planck }}$ gauge couplings are all close to $g_{i} \sim 0.5$, while $y_{t} \sim 0.35$ and $\lambda \sim 0.1$.

As shown in Fig. 2, the masses stay bounded up to the transition point to the symmetric phase, discussed in the next section. In the broken phase the effective mass relevant for the high energy behavior, obtained by rescaling all the momenta of the process $\left\{p_{i}\right\} \rightarrow\left\{\kappa p_{i}\right\} \kappa \rightarrow \infty$, up to an overall factor 7 is $m(\kappa) / \kappa \rightarrow 0$.

What is interesting is that the hierarchy of the effective masses gets mixed up. While the effective Higgs mass $m_{H}$ and the related Higgs potential mass $m$ are weakly scale dependent, the Higgs coupling $\lambda$ drops pretty fast by a factor about 8 , together causing the Higgs VEV $v=\sqrt{3 / \lambda} m_{H}$ to increase by a factor about 3.5. Note that, according to the mass-coupling relationships (23), what compares to the other couplings is $\sqrt{\lambda}$ not $\lambda$ itself. Given that $m_{H}$ is weakly scale dependent, what determines the mass hierarchy are the relations

$$
\frac{m_{W}\left(\mu^{2}\right)}{m_{H}\left(\mu^{2}\right)}=\sqrt{\frac{3}{4} \frac{g^{2}\left(\mu^{2}\right)}{\lambda\left(\mu^{2}\right)}}, \frac{m_{Z}\left(\mu^{2}\right)}{m_{H}\left(\mu^{2}\right)}=\sqrt{\frac{3}{4} \frac{g^{2}\left(\mu^{2}\right)+g^{\prime 2}\left(\mu^{2}\right)}{\lambda\left(\mu^{2}\right)}}, \frac{m_{t}\left(\mu^{2}\right)}{m_{H}\left(\mu^{2}\right)}=\sqrt{\frac{3}{2} \frac{y_{t}^{2}\left(\mu^{2}\right)}{\lambda\left(\mu^{2}\right)}},
$$

which must hold in the broken phase. Since $g$ is decreasing while $g^{\prime}$ is increasing the $Z$ boson mass grows most and exceeds $m_{H}$ above about $8 \times 10^{4} \mathrm{GeV}$ and even $m_{t}$ above about $7 \times 10^{10} \mathrm{GeV}$. The $W$ boson mass exceeds $m_{H}$ above about $5 \times 10^{6} \mathrm{GeV}$. These crossing happen in the history of the universe some time after inflation, but long before processes like nucleosynthesis set in. Whether they play any special role in the evolution of the universe I don't know.

\section{The issue of quadratic divergences in the SM}

A discussion of the large scale behavior in the SM is incomplete if we disregard the problem of quadratic divergences and the related hierarchy problem. In contrast to

\footnotetext{
${ }^{7}$ To remind the reader: a vertex function of $n_{B}$ boson fields and $n_{\psi}$ conjugate pairs of Fermi fields in the Landau gauge satisfies the RG equation

$$
\left\{\mu \frac{\partial}{\partial \mu}+\beta \frac{\partial}{\partial g}+\gamma_{m} m \frac{\partial}{\partial m}-n_{A} \gamma_{A}-2 n_{\psi} \gamma_{\psi}\right\} \Gamma(\{p\}, g, m, \mu)=0,
$$

and is a homogeneous function of canonical dimension dim $\Gamma=4-n_{B}-3 n_{\psi}$ under rescaling of all dimensionful quantities including momenta, masses and the renormalization scale $\mu$. The RG solution then may be written in the form

$$
\begin{aligned}
\Gamma(\{\kappa p\}, g, m, \mu) & =\kappa^{\operatorname{dim} \Gamma} \Gamma\left(\{p\}, g(\kappa), \frac{m(\kappa)}{\kappa}, \frac{\mu}{\kappa}\right) \\
& =\kappa^{\operatorname{dim} \Gamma} z_{B}(g, \kappa)^{-n_{B}} z_{\psi}(g, \kappa)^{-2 n_{\psi}} \Gamma\left(\{p\}, g(\kappa), \frac{m(\kappa)}{\kappa}, \mu\right)
\end{aligned}
$$
}

describing the asymptotic high-energy behavior. The $z$-factors represent the anomalous dimensions. 
the dimensionless running couplings all mass renormalizations (except the photon) are affected by quadratic ( $H, W$ and $Z$ ) or linear divergences (fermions), which are related universally to the renormalization of the Higgs potential parameter $m^{2}$ in the symmetric phase or equivalently to the Higgs VEV $v$ in the broken phase. Standard $\overline{\mathrm{MS}}$ mass RG equations usually only take into account the logarithmic singularities remaining after "throwing away" quadratic or linear divergences. Note that this is possible in this way only in the purely perturbative $\overline{\mathrm{MS}}$ scheme, while with a more physical lattice regularization, which applies beyond perturbation theory, the quadratic divergences cannot be eliminated this way. In other words the hierarchy problem is a real problem as discussed recently in 24]. In terms of masses the leading one-loop Higgs mass counterterm is given in Eq. (1) [50] (see also [19]), modulo small lighter fermion contributions. The one-loop coefficient function $C_{1}$ may be written as

$$
C_{1}=2 \lambda+\frac{3}{2} g^{\prime 2}+\frac{9}{2} g^{2}-12 y_{t}^{2}
$$

and is uniquely determined by dimensionless couplings. Surprisingly, taking into account the running of the SM couplings, which are not affected by quadratic divergences such that standard RG equations apply, the coefficient of the quadratic divergences of the Higgs mass counterterm vanishes at about $\mu_{0} \sim 7 \times 10^{16} \mathrm{GeV}$. As shown in [24] the next-order correction

$$
\begin{aligned}
C_{2}= & C_{1}+\frac{\ln \left(2^{6} / 3^{3}\right)}{16 \pi^{2}}\left[18 y_{t}^{4}+y_{t}^{2}\left(-\frac{7}{6} g^{\prime 2}+\frac{9}{2} g^{2}-32 g_{s}^{2}\right)\right. \\
& \left.+\frac{77}{8} g^{\prime 4}+\frac{243}{8} g^{4}+\lambda\left(-6 y_{t}^{2}+g^{\prime 2}+3 g^{2}\right)-\frac{10}{18} \lambda^{2}\right]
\end{aligned}
$$

does not change significantly this result. The same result applies for the Higgs potential parameter $m^{2}$ which corresponds to $m^{2} \hat{=} \frac{1}{2} M_{H}^{2}$. For scales $\mu<\mu_{0}$ we have $\delta m^{2}$ large negative, and thus triggering spontaneous symmetry breaking by a negative bare mass $m_{0}^{2}=m_{r}^{2}+\delta m^{2}$, where $m_{r}$ denotes a renormalized mass, while at $\mu=\mu_{0}$ the sign of $\delta m^{2}$ flips implying a phase transition to the symmetric phase at times $\sim 0.23 \times$ $10^{-38}$ to $10^{-42}$ seconds after the big bang. Such a transition is relevant for inflation scenarios in the evolution of the universe. At $\mu_{0}$ the Higgs VEV jumps to zero and SM gauge boson and fermion masses all vanish, at least provided the scalar self-coupling $\lambda$ continues to be positive. Note that the phase transition scale $\mu_{0}$ is close to the zero $\mu_{\lambda} \sim 3.5 \times 10^{17}$ of $\beta_{\lambda}$, i.e. $\beta_{\lambda}\left(\mu_{\lambda}\right)=0$. While $\lambda$ is decreasing below $\mu_{\lambda}$ it starts to increase weakly above that scale.

The important point is that to all orders of perturbation theory as well as beyond perturbation theory there exists a solution $C=0$, i.e. the relation Eq. (1) gets corrections by higher-order effects. Such relations are relations between the dimensionless gauge, Yukawa and Higgs couplings and do not depend on dimensionful low-energy parameters like the Higgs potential mass $m$ (in the symmetric phase) or the Higgs VEV $v$ (in the broken phase), which only show up as overall factors as the cutoff $\Lambda^{2}$ itself. Given all masses of the SM we note that $4 M_{t}^{2}>M_{H}^{2}+M_{Z}^{2}+2 M_{W}^{2}$ which makes the Higgs mass counterterm $\delta M_{H}^{2}<0$ and it is the heavy top which triggers spontaneous symmetry breaking in the $\mathrm{SM}$ as the bare mass square $M_{H 0}^{2}=M_{H}^{2}+\delta M_{H}^{2}$ is driven to negative values. Actually the quadratic divergence coefficient is dominated by the 


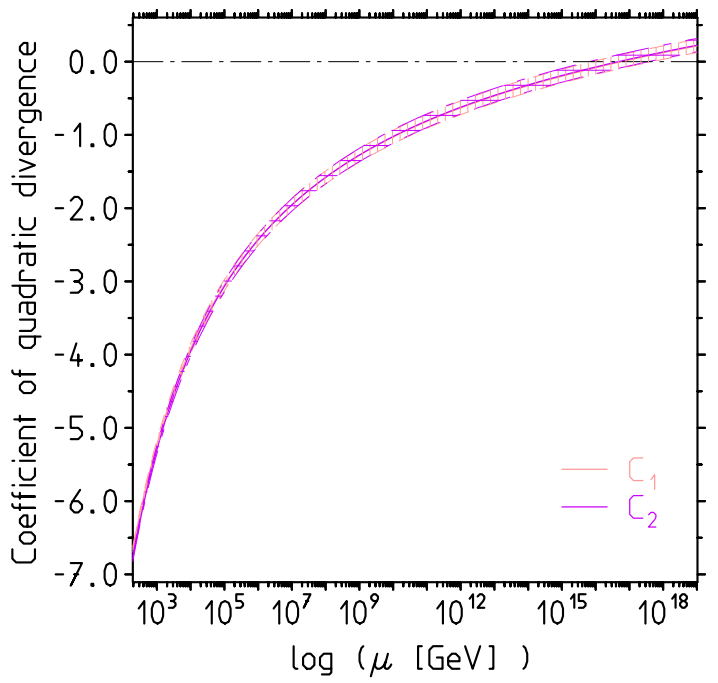

Figure 3: The coefficient of the quadratic divergence term at one and two loops as a function of the renormalization scale. The one-loop result essentially determines the behavior. The coefficient exhibits a zero, for $M_{H}=125 \mathrm{GeV}$ at about $\mu_{0} \sim 7 \times 10^{16}$, not far below $\mu=M_{\text {Planck }}$. The shaded band shows the parameter uncertainties given in Eqs. (21) 22 ).

leading order term and as a function of the $\overline{\mathrm{MS}}$ running couplings vanishes and changes sign below the Planck scale, as shown in Fig. 3. Interestingly, the top mass as well as the Higgs mass are such that the quadratic divergence vanished precisely not far below the Planck scale, as illustrated in Fig. 4. This observation has been pointed out recently in [24]. This does not really solve the hierarchy problem of the SM, of course as illustrated in Fig. 5. The scale dependence of the mass counterterm still is quadratic in the cutoff. Apparently there is a lack of bosonic contribution for canceling the big top mass contribution. In any case at the zero of the coefficient function there is a phase transition, which corresponds to a restoration of the symmetry. Such transition would take place at a scale $\mu \sim 10^{16}$ to $10^{18}$ one to three orders of magnitude below the Planck scale, at cosmic times $\sim 0.23 \times 10^{-38}$ to $10^{-42}$ sec and could have triggered inflation. Note that at the zero of $\beta_{\lambda}$ at about $\mu_{\lambda} \sim 3.5 \times 10^{17}>\mu_{0}$ the Higgs self-coupling $\lambda$ although rather small is still positive and then starts slowly increasing up to $M_{\text {Planck }}$.

If we take renormalization as a physical process similar to what it is condensed matter physics where both bare and renormalized (effective) quantities are physical, i.e. accessible to experiments, the key question is what happens to the effective Higgs potential $V=\frac{m^{2}}{2} H^{2}+\frac{\lambda}{24} H^{4}$. When the $m^{2}$ term changes sign and $\lambda$ stays positive, we know it is a first order phase transition. The vacuum jumps from $v=0$ to $v \neq 0$. Since the bare Lagrangian is the true Lagrangian (renormalization is just reshuffling terms) the change in sign of the bare mass is what determines the phase 8 .

\footnotetext{
${ }^{8}$ In the broken phase we have the mass coupling relations (28), which also must hold for the bare parameters. These relations then tell us that linear (fermion masses) and quadratic (boson masses) divergences are absent at the transition point. Except for $m$ all other masses vanish in the symmetric phase.
} 

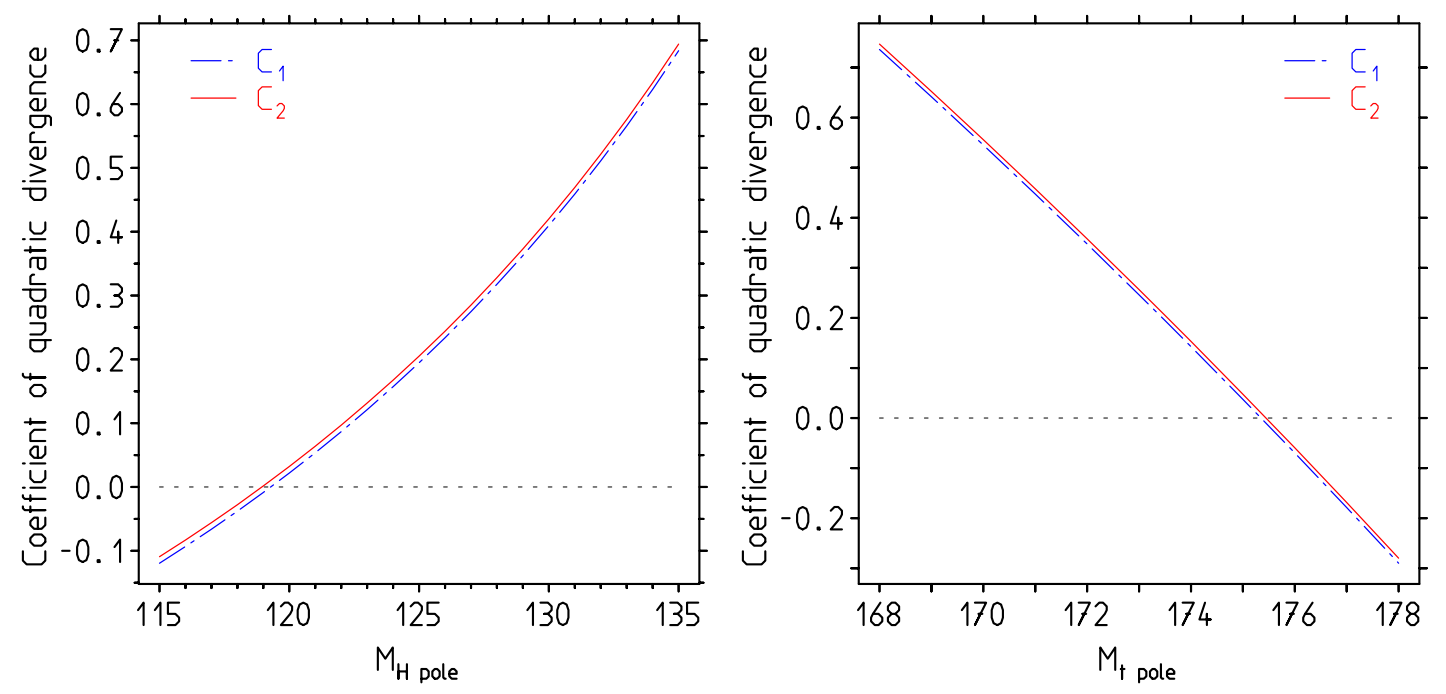

Figure 4: Left: the coefficient of the quadratic divergence term at $\mu=M_{\text {Planck }}$ as a function of $M_{H}$. Right: the same as a function of $M_{t}$.

Let us finally consider the possibilities of other phase changes. The crucial point is that the running of $v(\mu)$ is determined by the anomalous dimension of the Higgs potential parameter $m^{2}$ and by the $\beta$-function related to the renormalization of $\lambda$. Its behavior has been investigated recently in [21]. For high energies the second term of (24) is dominating, such that

$$
\mu^{2} \frac{d}{d \mu^{2}} \ln v^{2}\left(\mu^{2}\right) \sim-\frac{\beta_{\lambda}(\mu)}{\lambda(\mu)} .
$$

The behavior of $\lambda(\mu)$ and $\beta_{\lambda}(\mu)$ has been studied recently in the context of vacuum stability of the SM Higgs sector in Refs. [5-10] and reveals that the beta function $\beta_{\lambda}$ is negative up to a scale of about $3.5 \times 10^{17} \mathrm{GeV}$, where it changes sign. As already mentioned, above the zero $\mu_{\lambda}$ of $\beta_{\lambda}$ the effective coupling starts to increase again and the key question is whether at the zero of the beta function the effective coupling is still positive. In the latter case it will remain positive although small up to the Planck scale. In any case, at moderately high scales where $\beta_{\lambda}<0$, and provided $\lambda$ is still positive the following behavior is valid for the the Higgs VEV:

$$
\left.v^{2}\left(\mu^{2}\right)\right|_{\mu^{2} \rightarrow \infty} \sim\left(\mu^{2}\right)^{-\frac{\beta_{\lambda}(\mu)}{\lambda(\mu)}} \rightarrow \infty
$$

which means the $v^{2}\left(\mu^{2}\right)$ is increasing at these scales (where $\beta_{\lambda}<0$ and $\lambda>0$ ). The analyses [6] find that $\lambda$ turns negative (unstable or meta-stable Higgs potential) before the beta function reaches its zero. This may happen at rather low scales around $10^{10} \mathrm{GeV}$. In this case we would get an infinite Higgs VEV far below the Planck scale as an essential singularity. According to our analysis $\lambda$ remains positive up to the zero of the beta function and as a consequence up to the Planck scale in agreement with Refs. [5, 7, 21]. 

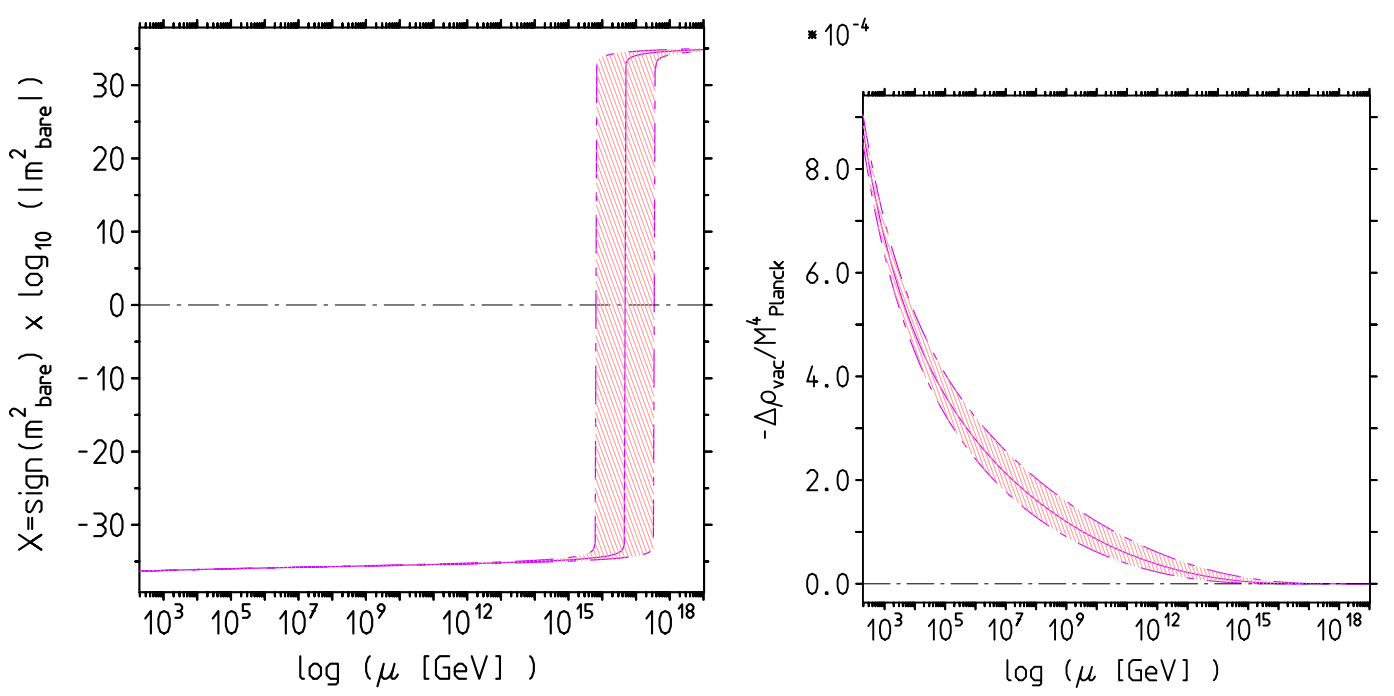

Figure 5: The EW phase transition in the SM. Left: $\operatorname{shown}$ is $X=\operatorname{sign}\left(m_{\text {bare }}^{2}\right) \times \log _{10}\left(\left|m_{\text {bare }}^{2}\right|\right)$ which represents $m_{\text {bare }}^{2}=\operatorname{sign}\left(m_{\text {bare }}^{2}\right) \times 10^{X}$. In the broken phase $m_{\text {bare }}^{2}=\frac{1}{2} m_{H \text { bare }}^{2}$. At the zero of the coefficient function shown in Fig. 3 the counterterm $\delta m^{2}=m_{\text {bare }}^{2}-m^{2}=0(m$ the $\overline{\mathrm{MS}}$ mass) vanishes and the bare mass changes sign. This represents a phase transition which triggers the Higgs mechanism and likely plays an important role for cosmic inflation. The band represents the parameter uncertainties Eqs. (21,22). Right: the "jump" $-\Delta \rho_{\mathrm{vac}}=$ $\frac{\lambda}{24} v_{\text {bare }}^{4}$ in units of $M_{\text {Planck }}^{4}$. At the transition point $\mu_{0}$ we have $v_{\text {bare }}=v\left(\mu_{0}^{2}\right)$, where $v\left(\mu^{2}\right)$ is the $\overline{\mathrm{MS}}$ renormalized VEV. The jump, too small to be seen in this plot, thus agrees with the renormalized one: $-\Delta \rho_{\mathrm{vac}}=\frac{\lambda\left(\mu_{0}^{2}\right)}{24} v^{4}\left(\mu_{0}^{2}\right)$, and thus is $O\left(v^{4}\right)$ and $\operatorname{not} O\left(M_{\text {Planck }}^{4}\right)$.

There are other phase transitions related to the parameter $\lambda . \beta_{\lambda}\left(\mu_{\lambda}\right)=0$ reveals that $\mu=\mu_{\lambda}$ is a second order phase transition form the antiscreening to the screening phase. Below $\mu_{\lambda}$ the coupling $\lambda$ is decreasing, while at $\mu_{\lambda}$ it starts to rise up to the Planck scale where the unknown cutoff theory is sitting and continuum field theory considerations break down. It is interesting to note that $\beta_{\lambda}(\mu)<0$ for $\mu<\mu_{\lambda}$ is due to the $S U(3)_{c}$ strong interaction contribution, the electroweak contribution alone is positive at all scales.

A very special phase transition may happen if $\lambda\left(\mu^{2}\right)$, decreasing for $\mu<\mu_{\lambda}$ gets zero. A vanishing $\lambda$ is a phase transition again by the fact that the potential changes shape; within the renormalizable SM $\lambda=0$ in the broken phase (where $m^{2}<0$ ) is an essential singularity, either the system gets unstable (i.e. non-nonsensical) or one has to remember that the SM is an effective theory only and at higher scales nonrenormalizable operators must come into play i.e a $\frac{\xi}{6 !} H^{6}$ term with positive coupling $\xi$, which keeps the system stable. Then one has three parameters in the relevant part of the potential $m^{2}, \lambda$ and $\xi$ and one may have more complicated vacuum structure, with metastable states etc. In other words the case $\lambda<0$ cannot be discussed without extending the SM. It requires to consider tri-critical system like the one encountered for superfluid $\mathrm{He}^{3}-\mathrm{He}^{4}$ mixtures. There a tri-critical point is the end point of a continuous line of second order phase transitions. 
If $\lambda=0$ before $\beta_{\lambda}=0$ the SM cannot be valid beyond that point $\mu^{*}$ where $\lambda\left(\mu^{*}\right)=0$. Note that in a renormalizable theory renormalization does not induce nonrenormalizable higher-order terms, so one really has to give up the SM in its literal form. Considered as a low energy effective tail of a condensed matter type system we anyway expect to see new structure at short distances as we have emphasized in the introduction.

\section{Remark on the impact on inflation}

As the Higgs system persists to make sense back to times of the early universe, it is attractive to think that the SM Higgs field itself is responsible for the inflation era of the early universe, as originally thought by Guth [51] (see also [52 55], [56] and references therein). The "inflation term", which comes in via the SM energy-momentum tensor, adds to the r.h.s of the Friedmann equation

$$
\ell^{2}\left(V(\phi)+\frac{1}{2} \dot{\phi}^{2}\right)
$$

where $\ell^{2}=8 \pi G / 3 . \quad M_{\mathrm{Pl}}=(G)^{-1 / 2}$ is the Planck mass, $G$ is Newton's gravitational constant and dots denote time derivatives. Inflation requires an exponential growth $a(t) \propto \mathrm{e}^{H t}$ of the Friedman radius $a(t)$ of the universe, where $H(t)=\dot{a} / a(t)$ is the Hubble constant at cosmic time $t$.

The contribution of the Higgs to the energy momentum tensor amounts to a contribution to energy density and pressure given by

$$
\rho_{\phi}=\frac{1}{2} \dot{\phi}^{2}+V(\phi) ; p_{\phi}=\frac{1}{2} \dot{\phi}^{2}-V(\phi) .
$$

The second Friedman equation 9 reads $\ddot{a} / a=-\frac{\ell^{2}}{2}(\rho+3 p)$ and the condition for growth is $\ddot{a}>0$ which requires $p<-\rho / 3$ and hence $\frac{1}{2} \dot{\phi}^{2}<V(\phi)$. The first Friedman equation $\operatorname{reads} \dot{a}^{2} / a^{2}+k / a^{2}=\ell^{2} \rho$ and may be written as $H^{2}=\ell^{2}\left[V(\phi)+\frac{1}{2} \dot{\phi}^{2}\right]=\ell^{2} \rho$. The field equation reads $\ddot{\phi}+3 H \dot{\phi}=-V^{\prime}(\phi)$ and the kinetic term $\dot{\phi}^{2}$ is controlled by $\dot{H}=-\frac{3}{2} \ell^{2} \dot{\phi}^{2}$ related to the observationally controlled deceleration parameter $q(t)=$ $-\ddot{a} a / \dot{a}^{2}$. By inflation $k / a^{2}(t) \rightarrow 0(k=0, \pm 1$ the normalized curvature $)$, such that the universe looks effectively flat $(k=0)$ for any initial $k$. Inflation looks to be universal for quasi-static fields $\dot{\phi} \sim 0$ and $V(\phi)$ large positive. Then $a(t) \propto \exp (H t)$ with $H \simeq \ell \sqrt{V(\phi)}$. This is precisely what the transition to the symmetric phase suggests. Now, as for the Higgs potential $\lambda$ remains positive and the bare mass square also has been positive (symmetric phase) before it flipped to negative values at later times, this definitely supports the inflation condition. As both $\lambda$ and $m^{2}$ for the first time are numerically fairly well known quantitative conclusions on the inflation patterns should be possible solely on the basis of SM properties. The leading behavior is characterized by a free massive scalar field with potential $V=\frac{m^{2}}{2} \phi^{2}$, such that $H^{2}=(\dot{a} / a)^{2}=\frac{m^{2}}{6} \phi^{2}$ and $\ddot{\phi}+3 \dot{H}(\phi)=m^{2} \phi$ which is nothing but a harmonic oscillator

\footnotetext{
${ }^{9}$ We drop the cosmological constant term which plays a role only at larger times.
} 
with friction. A constant background field $\phi \rightarrow \phi_{0}+\phi$ would imply a dark energy term (cosmological constant) of the right sign. In contrast after the phase transition triggered by the change of sign in the bare $m^{2}$ the scalar VEV implies a cosmological constant contribution $-\frac{\lambda}{24} v^{4}$ of negative sign 10 .

The cosmological constant and the missing cold dark matter problems still persist. The scenario suggested by the present analysis is a Gaussian potential with small anharmonic perturbations, since $m^{2}$ is predicted to be large while $\lambda$ remains small. This picture should be valid in the renormalizable effective field theory regime below about $10^{17} \mathrm{GeV}$. Going to higher energies details of the cutoff system are expected to come into play, effectively in form of dimension 5 and/or dimension 6 operators as leading corrections. These corrections are expected to get relevant only closer to the Planck scale.

\section{Conclusion}

We adopt that the new particle found by ATLAS and CMS at the LHC is the SM Higgs and we argue about the specific value found for the Higgs mass $M_{H}=125.5 \pm 1.5 \mathrm{GeV}$ and its impact for the SM itself. We understand the SM as a low energy effective emergence of some unknown physical system, we may call it "ether", which is located at the Planck scale with the Planck length as a "microscopic" length scale. Note that the cutoff, though very large, in any case is finite. Correspondingly, counterterms are finite. As an example, $\delta M_{H}^{2}=\frac{M_{\text {Planck }}^{2}}{16 \pi^{2}} C_{2}\left(\mu=M_{\text {Planck }}\right)$, with $C_{2}\left(\mu=M_{\text {Planck }}\right) \sim 0.2$, where $C_{2}$ is the coefficient of the quadratic divergence of the bare Higgs mass given in Eqs. (229). As noted quite some time ago in Ref. [3] stability of the SM vacuum up to the Planck scale is just what it now turns out to be and this is far form being an accident. It signals a higher self-consistency of the SM than anticipated before. The Higgs potential remains stable, there is no non-perturbative Higgs issue, no Landau pole or whatever. The crucial thing is that this is the result of the a conspiracy of the several interactions "unified" in the SM which lead to this result, top Yukawa, strong interaction as well as the gauge couplings turn out to be crucial for the stability of the SM ground state. Note that the full knowledge of the RG coefficients is needed to to obtain a stable solution, while approximations like the gaugeless one may suggest a false metastable situation. In this kind of low energy effective scenario vacuum fluctuations as well as the issue of quadratic divergences unavoidably have to be taken into consideration. In this paper we have reconsidered the issue of quadratic divergences and observe that the zero in the corresponding coefficient, reported recently in Ref. [24], implies a first order phase transition at about $\mu_{0} \sim 7 \times 10^{16} \mathrm{GeV}$ above which the system is in the unbroken phase i.e. the Higgs VEV is vanishing and all modes besides the

${ }^{10}$ The chiral phase transition of QCD which leads to quark condensates contributing

$$
T_{\mu \nu \mathrm{QCD}, \mathrm{vac}}=-\left\langle 0\left|\mathcal{L}_{\mathrm{QCD}}\right| 0\right\rangle g_{\mu \nu}=\left\{m_{u} \bar{u} u+m_{d} \bar{d} d+m_{s} \bar{s} s+\cdots\right\} g_{\mu \nu}
$$

to the cosmological constant, has to be reconsidered under the aspect that the relation between bare an renormalized quantities are physical in the low energy effective approach. Maybe it is possible to give a more precise meaning also to the gluon condensate within this context. 
remaining complex Higgs doublet fields are massless and thus ultrarelativistic. Above the transition point the number of massless degrees of freedom (radiation) of the SM consists of $g_{f}=90$ fermionic degrees of freedom and $g_{B}=24$ bosonic ones such that the effective number of degrees of freedom $g_{*}(T)=g_{B}(T)+\frac{7}{8} g_{f}(T)=102.75$ (the factor $\frac{7}{8}$ accounts for the Pauli exclusion principle which applies for the fermions). The four Higgses in the symmetric phase have equal masses, which likely would be heavy, not necessarily relativistic. If all SM modes would be massless we would have $g_{*}(T)=$ 106.75, which effectively applies for temperatures large relative to about $2 M_{\max }<$ $500 \mathrm{GeV}$, where $M_{\max }$ is the upper bound for all running SM masses in the range up to the transition point (see Fig. 2). Below the transition point we know that the one remaining physical Higgs is as light as the other SM particles11. Our analysis shows that it need not be true that the higher the energy the more relativistic degrees of freedom must show up. The EW phase transition thus allows for very heavy Higgses in the symmetric phase while the light Higgs in the broken phase is a consequence of the phase transition itself, e.g. if the bare Higgs mass is of the order of $M_{\text {Planck }}$ which gets moderately $\left(C_{2}\left(\mu=M_{\text {Planck }}\right) \sim+0.2\right)$ enhanced by radiative corrections in the symmetric phase while it compensates the bare mass in the broken phase (at the EW scale $\left.C_{2}(\mu=v) \sim-6.6\right)$. Note that in our scenario the EW phase transition takes place at $\mu_{0} \sim 7 \times 10^{16} \mathrm{GeV}$ not near $\mu \sim v \simeq 246.22 \mathrm{GeV}$. This has an impact on baryogenesis, which has to be reconsidered given the new insight. In particular, using available 3-loop RG equations is crucial for getting the proper answer. Our approach sheds new light on the problem of the EW phase transition and baryogenesis since commonly accepted assumptions (see e.g. [57,58]) have to be reconsidered. With the now known SM parameters and taking into account presently known higher order perturbative result a new picture may emerge. Without affecting the basic symmetry structure of the SM, the latter enhanced by very heavy singlet Majorana neutrinos not only would provide the most natural explanation for the smallness of the neutrino masses by the resulting sea-saw mechanism, but eventually explain dark matter and play a role to explain the observed baryon asymmetry.

So far our scenario does not say anything about cold dark matter. However, also this issue has to be thought newly. If the EW phase transition is so much higher up domains of symmetric phases could have formed. The physics of the symmetric phase is very different form the one in the broken phase. The heavy Higgses would decay into massless fermions, at leading order not into gauge bosons, as e.g. $\Gamma(H \rightarrow W W)$ vanishes in the limit $M_{W} \rightarrow 0$, while $\Gamma(H \rightarrow \bar{f} f)=\frac{M_{H} y_{f}^{2}}{16 \pi}$ for massless fermions. Preferred are the modes with larger Yukawa coupling as in the broken phase. The "charged" Higgses, physical in the symmetric phase, decay parity violating like $\phi^{+} \rightarrow$ $\tau^{+} \nu_{\tau}$ for example, however an electric charge as such in only defined after symmetry breaking. This may be another interesting aspect: in the symmetric phase there are no electromagnetic interactions and $S U(2)_{L}$ is unbroken in the very early phase of the universe. In the symmetric phase there could exist heavy $S U(2)$ bound states which would bind energy and could be dark matter candidates. Thus cold dark matter could be frozen energy, very similar to ordinary matter, which is mainly hadronic binding energy (nucleon masses), while the masses of the elementary fields induced by the

${ }^{11} \mathrm{SM}$ particles contribute $\rho_{\mathrm{rad}}(T)=\frac{\pi^{2}}{30} g_{*}(T) T^{4}$ to the radiation density. 
Higgs mechanism constitute an almost negligible contribution to normal matter in the universe. The way matter clusters and populates the universe of course is determined by the details of the Yukawa- and Higgs-sector and the particular form of the EW phase transition.

Across the phase transition also the cosmological constant undergoes a dramatic jump by $\Delta \rho_{\text {vac }} \doteq \rho_{\text {vac, broken }}-\rho_{\text {vac, symmetric }}=-\frac{\lambda}{24} v^{4}$ as the Higgs VEV generated contributes a huge negative term $\rho_{\mathrm{vac}}(v)=-\frac{\lambda}{24} v^{4}$ absent in the symmetric phas 12 . The vacuum energy $V(0)$ in the symmetric phase is expected to be a function of $M_{\text {Planck }}$, naturally $\langle V(0)\rangle \propto M_{\text {Planck }}^{4}$, and the Higgs parameter $m$ of the almost Gaussian SM Higgs potential $V(\Phi)=m^{2} \Phi^{+} \Phi+\frac{\lambda}{3 !}\left(\Phi^{+} \Phi\right)^{2} \simeq m^{2} \Phi^{+} \Phi$, while the dependence on the Higgs interaction term is small. Here $\Phi$ is the complex SM Higgs doublet field, which in the symmetric phase includes four physical scalars. Note that gravity as we see it at long distances in our scenario emerges form the "ether" system exhibiting an intrinsic fundamental cutoff. Therefore, there is no reason why we should expect gravity to to be quantized in the sense of e local relativistic renormalizable QFT at the Planck scale. Also for gravity the low energy manifestation is expected to be what is obtained form a low energy expansion (see Ref. [18] for a corresponding consideration).

Our mains conclusions are the following: with the determination of the Higgs mass all relevant parameters of the SM are known. Together with recent progress in the calculation of higher order RG coefficient functions and matching conditions, perturbative RG evolution of SM parameters up to the Planck scale turns out the apply, without encountering singularities like Landau poles. Perturbative methods thus allows one to control SM physics up to scales not far below the Planck scale, where the effective approach breaks down. Key point is that the SM Higgs potential remains stable up to the Planck scale. Surprisingly, the Higgs mechanism is triggered by the SM itself, by the fact that the bare mass parameter in the Higgs potential changes sign at about $10^{17} \mathrm{GeV}$ not far below the Plank scale. Accordingly, the EW phase transition has taken place much earlier in the history of the universe than anticipated usually. Before the EW phase transition took place the symmetric phase of the SM exhibiting a rather different physics profile must have played an important role in the early universe. Its consequences have to be worked out yet. Our findings also shed new light on the inflation epoch in the early universe.

\section{Acknowledgments:}

I am grateful to Mikhail Kalmykov for many inspiring discussions, for critically reading the manuscript as well as for long-time collaboration in electroweak two-loop calculations and in particular on working out the relationship between on-shell and $\overline{\mathrm{MS}}$ parameters in the SM, which play a key role in the present work. I also thank for support by the EC Program Transnational Access to Research Infrastructure (TARI) INFN - LNF, HadronPhysics3 - Integrating Activity, Contract No. 283286.

\footnotetext{
${ }^{12}$ At scale $\mu_{0} \sim 7 \times 10^{16}$ we have $\lambda \sim 0.115$ and $v \sim 695 \mathrm{GeV}$ such that $\frac{\lambda}{24} v^{4} \sim 1.1 \times 10^{9} \mathrm{GeV}^{4}$. Converted with the factor $\kappa=8 \pi G$, it corresponds to a shift $\Delta \Lambda_{\mathrm{EW}}=\kappa \Delta \rho_{\mathrm{vac}} \simeq-0.5 \mathrm{~cm}^{-2}$ in the cosmological constant $\Lambda$, while the observed value is given by $\Lambda_{\text {obs }}=\kappa \rho_{\text {crit }} \Omega_{\Lambda}=1.6517 \times 10^{-56} \mathrm{~cm}^{-2}$. $\Omega_{\Lambda}=0.67_{-0.023}^{+0.027}\left[56\right.$ is the dark energy fraction of the critical energy density $\rho_{\text {crit }}=3 H_{0}^{2} \kappa^{-1}=1.878 \times$ $10^{-29} h^{2} \mathrm{gr} / \mathrm{cm}^{3}$ with $h=0.67 \pm 0.02$ for which the universe is flat. A mechanism to tame contributions to the dark energy is still not known to my knowledge (see e.g. [59]).
} 


\section{Appendix: the ingredients for one-loop matching}

The one-loop on-shell counterterms may be expressed in terms of the known scalar integrals:

$$
\begin{aligned}
A_{0}(m) & =-m^{2}\left(\operatorname{Reg}+1-\ln m^{2}\right) \\
B_{0}\left(m_{1}, m_{2} ; s\right) & =\operatorname{Reg}-\int_{0}^{1} \mathrm{~d} z \ln \left(-s z(1-z)+m_{1}^{2}(1-z)+m_{2}^{2} z-\mathrm{i} \varepsilon\right),
\end{aligned}
$$

with

$$
\operatorname{Reg}=\frac{2}{\varepsilon}-\gamma+\ln 4 \pi+\ln \mu_{0}^{2} \equiv \ln \mu^{2} .
$$

In addition we define $\mathcal{C}=\frac{\sqrt{2} G_{\mu}}{16 \pi^{2}}$ and $\mathcal{C}_{\mu}=\frac{\sqrt{2} G_{\mu}}{16 \pi^{2}} \ln \mu^{2}$. Furthermore, $c_{W}^{2}=\frac{M_{W}^{2}}{M_{Z}^{2}}$ and $s_{W}^{2}=1-c_{W}^{2}$. Sums over fermion contributions we write $\sum_{f_{s}}$ for sums over individual fermions, and $\sum_{f_{d}}$ for sums over fermion doublets. $Q_{f}$ denotes the fermion charge, $a_{f}=Q_{f} s_{W}^{2} \mp \frac{1}{4}$ the $Z f \bar{f}$ vector coupling and by $b_{f}= \pm \frac{1}{4}$ the axialvector couplings. A color factor $N_{c}=3$ applies for quarks.

$\overline{\mathrm{MS}}$ counterterms:

$$
\begin{aligned}
& \left.\frac{\delta v^{-1}}{v^{-1}}\right|_{\overline{\mathrm{MS}}}=+\mathcal{C}_{\mu}\left(3 \frac{M_{Z}^{4}}{M_{H}^{2}}+6 \frac{M_{W}^{4}}{M_{H}^{2}}-\frac{3}{2} M_{Z}^{2}-3 M_{W}^{2}+\frac{3}{2} M_{H}^{2}\right. \\
& \left.+\sum_{f_{d}}\left[-4 \frac{m_{1}^{2}+m_{2}^{2}}{M_{H}^{2}}+m_{1}^{2}+m_{2}^{2}\right]\right) \\
& \left.\frac{\delta M_{Z}^{2}}{M_{Z}^{2}}\right|_{\overline{\mathrm{MS}}}=+\mathcal{C}_{\mu}\left(-6 \frac{M_{Z}^{4}}{M_{H}^{2}}-12 \frac{M_{W}^{4}}{M_{H}^{2}}+\frac{11}{3} M_{Z}^{2}+\frac{14}{3} M_{W}^{2}-28 c_{W}^{2} M_{W}^{2}-3 M_{H}^{2}\right. \\
& \left.+\sum_{f_{s}}\left[8 \frac{m_{f}^{4}}{M_{H}^{2}}-2 m_{f}^{2}+\frac{22}{27}\left(M_{Z}^{2}-2 M_{W}^{2}\right)+\frac{40}{27} c_{W}^{2} M_{W}^{2}\right]\right) \\
& \left.\frac{\delta M_{W}^{2}}{M_{W}^{2}}\right|_{\overline{\mathrm{MS}}}=+\mathcal{C}_{\mu}\left(-6 \frac{M_{Z}^{4}}{M_{H}^{2}}-12 \frac{M_{W}^{4}}{M_{H}^{2}}+3 M_{Z}^{2}-\frac{68}{3} M_{W}^{2}-3 M_{H}^{2}\right. \\
& \left.+\sum_{f_{d}}\left[8 \frac{m_{1}^{4}+m_{2}^{4}}{M_{H}^{2}}-2\left(m_{1}^{2}+m_{2}^{2}\right)+\frac{4}{3} M_{W}^{2}\right]\right) \\
& \left.\frac{\delta M_{H}^{2}}{M_{H}^{2}}\right|_{\overline{\mathrm{MS}}}=+\mathcal{C}_{\mu}\left(-3 M_{Z}^{2}-6 M_{W}^{2}+3 M_{H}^{2}+\sum_{f_{s}}\left[2 m_{f}^{2}\right]\right) \\
& \left.\frac{\delta m_{t}}{m_{t}}\right|_{\overline{\mathrm{MS}}}=+\mathcal{C}_{\mu}\left(12 \frac{M_{t}^{4}}{M_{H}^{2}}+12 \frac{M_{b}^{4}}{M_{H}^{2}}-3 \frac{M_{Z}^{4}}{M_{H}^{2}}-6 \frac{M_{W}^{4}}{M_{H}^{2}}-\frac{3}{2} M_{b}^{2}+\frac{3}{2} M_{t}^{2}-\frac{4}{3} M_{Z}^{2}\right. \\
& \left.+\frac{20}{3} M_{W}^{2}-\frac{16}{3} c_{W}^{2} M_{W}^{2}-\frac{3}{2} M_{H}^{2}-\frac{16}{3} c_{W}^{2} s_{W}^{2} M_{Z}^{2}\right) \\
& \left.\frac{\delta m_{b}}{m_{b}}\right|_{\overline{\mathrm{MS}}}=+\mathcal{C}_{\mu}\left(12 \frac{M_{t}^{4}}{M_{H}^{2}}+12 \frac{M_{b}^{4}}{M_{H}^{2}}-3 \frac{M_{Z}^{4}}{M_{H}^{2}}-6 \frac{M_{W}^{4}}{M_{H}^{2}}+\frac{3}{2} M_{b}^{2}-\frac{3}{2} M_{t}^{2}+\frac{2}{3} M_{Z}^{2}\right. \\
& \left.+\frac{2}{3} M_{W}^{2}-\frac{4}{3} c_{W}^{2} M_{W}^{2}-\frac{3}{2} M_{H}^{2}-\frac{4}{3} c_{W}^{2} s_{W}^{2} M_{Z}^{2}\right)
\end{aligned}
$$


On-shell counterterms:

$$
\begin{aligned}
& \frac{\delta e}{e}=\mathcal{C} s_{W}^{2} M_{W}^{2}\left(\frac{38}{3}+14 \frac{A_{0}\left(M_{W}\right)}{M_{W}^{2}}-\frac{8}{3} \sum_{f_{s}} Q_{f}^{2}\left(1+\frac{A_{0}\left(m_{f}\right)}{m_{f}^{2}}\right)\right) \\
& \frac{\delta v^{-1}}{v^{-1}}=\frac{\delta e}{e}-\frac{1}{2 s_{W}^{2}}\left(s_{W}^{2} \frac{\delta M_{W}^{2}}{M_{W}^{2}}+c_{W}^{2} \frac{\delta M_{Z}^{2}}{M_{Z}^{2}}\right) \\
& \delta M_{H}^{2}=\mathcal{C}\left(A_{0}\left(M_{H}\right)\left(3 M_{H}^{2}\right)\right. \\
& +A_{0}\left(M_{Z}\right)\left(M_{H}^{2}+6 M_{Z}^{2}\right) \\
& +B_{0}\left(M_{H}, M_{H}, M_{H}^{2}\right)\left(\frac{9}{2} M_{H}^{4}\right) \\
& +B_{0}\left(M_{Z}, M_{Z}, M_{H}^{2}\right)\left(\frac{1}{2} M_{H}^{4}-2 M_{H}^{2} M_{Z}^{2}+6 M_{Z}^{4}\right) \\
& +A_{0}\left(M_{W}\right)\left(2 M_{H}^{2}+12 M_{W}^{2}\right) \\
& +B_{0}\left(M_{W}, M_{W}, M_{H}^{2}\right)\left(M_{H}^{4}-4 M_{H}^{2} M_{W}^{2}+12 M_{W}^{4}\right) \\
& \left.+\sum_{f_{s}}\left[A_{0}\left(m_{f}\right)\left(-8 m_{f}^{2}\right)+B_{0}\left(m_{f}, m_{f}, M_{H}^{2}\right)\left(2 M_{H}^{2} m_{f}^{2}-8 m_{f}^{4}\right)\right]\right) \\
& \delta M_{Z}^{2}=\mathcal{C}\left(-\frac{2}{3} M_{H}^{2} M_{Z}^{2}+4 \frac{M_{Z}^{4}}{M_{H}^{2}} M_{Z}^{2}-\frac{2}{9} M_{Z}^{4}\right. \\
& +8 \frac{M_{W}^{4}}{M_{H}^{2}} M_{Z}^{2}-\frac{20}{9} M_{W}^{2} M_{Z}^{2}+\frac{16}{3} M_{W}^{4}-16 c_{W}^{2} M_{W}^{4} \\
& +A_{0}\left(M_{H}\right)\left(\frac{1}{3} M_{H}^{2}+2 M_{Z}^{2}\right) \\
& +A_{0}\left(M_{Z}\right)\left(-\frac{1}{3} M_{H}^{2}+6 \frac{M_{Z}^{4}}{M_{H}^{2}}+\frac{2}{3} M_{Z}^{2}\right) \\
& +A_{0}\left(M_{W}\right)\left(12 \frac{M_{W}^{2}}{M_{H}^{2}} M_{Z}^{2}+\frac{16}{3} M_{W}^{2}-16 c_{W}^{2} M_{W}^{2}+\frac{2}{3} M_{Z}^{2}\right) \\
& +B_{0}\left(M_{Z}, M_{H}, M_{Z}^{2}\right)\left(-\frac{4}{3} M_{H}^{2} M_{Z}^{2}+\frac{1}{3} M_{H}^{4}+4 M_{Z}^{4}\right) \\
& +B_{0}\left(M_{W}, M_{W}, M_{Z}^{2}\right)\left(\frac{16}{3} M_{W}^{2} M_{Z}^{2}-\frac{68}{3} M_{W}^{4}-16 c_{W}^{2} M_{W}^{4}+\frac{1}{3} M_{Z}^{4}\right) \\
& +\sum_{f_{s}}\left[\left(\frac{32}{3} M_{Z}^{2} m_{f}^{2}-\frac{16}{9} M_{Z}^{4}\right)\left(a_{f}^{2}+b_{f}^{2}\right)\right. \\
& +A_{0}\left(m_{f}\right)\left(-8 \frac{M_{Z}^{2}}{M_{H}^{2}} m_{f}^{2}+\frac{32}{3} M_{Z}^{2}\left(a_{f}^{2}+b_{f}^{2}\right)\right) \\
& \left.\left.+\quad B_{0}\left(m_{f}, m_{f}, M_{Z}^{2}\right)\left(\frac{32}{3} M_{Z}^{2} m_{f}^{2}\left(a_{f}^{2}-2 b_{f}^{2}\right)+\frac{16}{3} M_{Z}^{4}\left(a_{f}^{2}+b_{f}^{2}\right)\right)\right]\right)
\end{aligned}
$$




$$
\begin{aligned}
& \delta M_{W}^{2}=\mathcal{C}\left(-\frac{2}{3} M_{H}^{2} M_{W}^{2}+8 \frac{M_{W}^{6}}{M_{H}^{2}}+4 \frac{M_{Z}^{4}}{M_{H}^{2}} M_{W}^{2}-\frac{112}{9} M_{W}^{4}-\frac{2}{3} M_{Z}^{2} M_{W}^{2}\right. \\
& +A_{0}\left(M_{H}\right)\left(2 M_{W}^{2}+\frac{1}{3} M_{H}^{2}\right) \\
& +A_{0}\left(M_{Z}\right)\left(6 \frac{M_{Z}^{2}}{M_{H}^{2}} M_{W}^{2}+\frac{8}{3} M_{W}^{2}-8 c_{W}^{2} M_{W}^{2}+\frac{1}{3} M_{Z}^{2}\right) \\
& +A_{0}\left(M_{W}\right)\left(12 \frac{M_{W}^{4}}{M_{H}^{2}}-4 M_{W}^{2}-\frac{1}{3} M_{H}^{2}-\frac{1}{3} M_{Z}^{2}\right) \\
& +B_{0}\left(M_{H}, M_{W}, M_{W}^{2}\right)\left(-\frac{4}{3} M_{H}^{2} M_{W}^{2}+\frac{1}{3} M_{H}^{4}+4 M_{W}^{4}\right) \\
& +B_{0}\left(M_{Z}, M_{W}, M_{W}^{2}\right)\left(-\frac{68}{3} M_{W}^{4}-16 c_{W}^{2} M_{W}^{4}+\frac{1}{3} M_{Z}^{4}+\frac{16}{3} M_{Z}^{2} M_{W}^{2}\right) \\
& +B_{0}\left(0, M_{W}, M_{W}^{2}\right)\left(-16 s_{W}^{2} M_{W}^{4}\right) \\
& +\sum_{f_{d}}\left[\frac{4}{3} M_{W}^{2}\left(m_{1}^{2}+m_{2}^{2}\right)-\frac{4}{9} M_{W}^{4}\right. \\
& +A_{0}\left(m_{1}\right)\left(-8 \frac{M_{W}^{2}}{M_{H}^{2}} m_{1}^{2}+\frac{4}{3} M_{W}^{2}-\frac{2}{3}\left(m_{1}^{2}-m_{2}^{2}\right)\right) \\
& +A_{0}\left(m_{2}\right)\left(-8 \frac{M_{W}^{2}}{M_{H}^{2}} m_{2}^{2}+\frac{4}{3} M_{W}^{2}+\frac{2}{3}\left(m_{1}^{2}-m_{2}^{2}\right)\right) \\
& \left.\left.+\quad B_{0}\left(m_{1}, m_{2}, M_{W}^{2}\right)\left(\frac{4}{3} M_{W}^{4}-\frac{2}{3} M_{W}^{2}\left(m_{1}^{2}+m_{2}^{2}\right)-\frac{2}{3}\left(m_{1}^{2}-m_{2}^{2}\right)^{2}\right)\right]\right) \\
& \delta m_{\tau}=m_{\tau} \mathcal{C}\left(4 \frac{M_{W}^{4}}{M_{H}^{2}}+2 \frac{M_{Z}^{4}}{M_{H}^{2}}-3 M_{W}^{2}+\frac{3}{2} M_{Z}^{2}\right. \\
& +A_{0}\left(M_{H}\right) \\
& +A_{0}\left(M_{Z}\right)\left(3 \frac{M_{Z}^{2}}{M_{H}^{2}}+6 \frac{M_{W}^{2}}{m_{\tau}^{2}}-4 c_{W}^{2} \frac{M_{W}^{2}}{m_{\tau}^{2}}-\frac{5}{2} \frac{M_{Z}^{2}}{m_{\tau}^{2}}\right) \\
& +A_{0}\left(M_{W}\right)\left(6 \frac{M_{W}^{2}}{M_{H}^{2}}-\frac{M_{W}^{2}}{m_{\tau}^{2}}+\frac{1}{2}\right) \\
& +A_{0}\left(m_{\tau}\right)\left(-2 \frac{M_{W}^{2}}{m_{\tau}^{2}}+\frac{5}{2} \frac{M_{Z}^{2}}{m_{\tau}^{2}}+1\right) \\
& +B_{0}\left(m_{\tau}, M_{H}, m_{\tau}^{2}\right)\left(-\frac{1}{2} M_{H}^{2}+2 m_{\tau}^{2}\right) \\
& +B_{0}\left(m_{\tau}, M_{Z}, m_{\tau}^{2}\right)\left(6 M_{W}^{2} M_{Z}^{2} / m_{\tau}^{2}+12 M_{W}^{2}-8 c_{W}^{2} M_{W}^{2}-4 \frac{M_{W}^{4}}{m_{\tau}^{2}}-\frac{7}{2} M_{Z}^{2}-\frac{5}{2} \frac{M_{Z}^{4}}{m_{\tau}^{2}}\right) \\
& +B_{0}\left(m_{\tau}, 0, m_{\tau}^{2}\right)\left(-8 M_{W}^{2}+8 c_{W}^{2} M_{W}^{2}\right) \\
& \left.+B_{0}\left(m_{\nu_{\tau}}, M_{W}, m_{\tau}^{2}\right)\left(\frac{1}{2} M_{W}^{2}-\frac{M_{W}^{4}}{m_{\tau}^{2}}+\frac{1}{2} m_{\tau}^{2}\right)+\sum_{f_{s}} A_{0}\left(m_{f}\right)\left(-4 \frac{m_{f}^{2}}{M_{H}^{2}}\right)\right)
\end{aligned}
$$




$$
\begin{aligned}
& \delta M_{b}=M_{b} \mathcal{C}\left(-\frac{4}{9} s_{W}^{2} M_{W}^{2}+2 \frac{M_{Z}^{4}}{M_{H}^{2}}+4 \frac{M_{W}^{4}}{M_{H}^{2}}-\frac{13}{18} M_{Z}^{2}-\frac{11}{9} M_{W}^{2}+\frac{4}{9} \frac{M_{W}^{4}}{M_{Z}^{2}}\right. \\
& +A_{0}\left(M_{H}\right) \\
& +A_{0}\left(M_{W}\right)\left(\frac{1}{2}+6 \frac{M_{W}^{2}}{M_{H}^{2}}-\frac{1}{2} \frac{M_{t}^{2}}{M_{b}^{2}}-\frac{M_{W}^{2}}{M_{b}^{2}}\right) \\
& +A_{0}\left(M_{Z}\right)\left(3 \frac{M_{Z}^{2}}{M_{H}^{2}}-\frac{5}{18} \frac{M_{Z}^{2}}{M_{b}^{2}}+\frac{2}{9} \frac{M_{W}^{2}}{M_{b}^{2}}-\frac{4}{9} \frac{M_{W}^{2}}{M_{Z}^{2}} \frac{M_{W}^{2}}{M_{b}^{2}}\right) \\
& +A_{0}\left(M_{t}\right)\left(\frac{1}{2}+\frac{1}{2} \frac{M_{t}^{2}}{M_{b}^{2}}+\frac{M_{W}^{2}}{M_{b}^{2}}\right) \\
& +A_{0}\left(M_{b}\right) 4 s_{W}^{2}\left(\frac{1}{3} \frac{M_{W}^{2}}{M_{b}^{2}}\right) \\
& +A_{0}\left(M_{b}\right)\left(1+\frac{5}{18} \frac{M_{Z}^{2}}{M_{b}^{2}}-\frac{2}{9} \frac{M_{W}^{2}}{M_{b}^{2}}+\frac{4}{9} \frac{M_{W}^{2}}{M_{Z}^{2}} \frac{M_{W}^{2}}{M_{b}^{2}}\right) \\
& +B_{0}\left(M_{t}, M_{W}, M_{b}^{2}\right)\left(\frac{1}{2} M_{b}^{2}-M_{t}^{2}+\frac{1}{2} \frac{M_{t}^{4}}{M_{b}^{2}}+\frac{1}{2} M_{W}^{2}+\frac{1}{2} M_{W}^{2} \frac{M_{t}^{2}}{M_{b}^{2}}-\frac{M_{W}^{4}}{M_{b}^{2}}\right) \\
& +B_{0}\left(M_{b}, M_{H}, M_{b}^{2}\right)\left(2 M_{b}^{2}-\frac{1}{2} M_{H}^{2}\right) \\
& +B_{0}\left(M_{b}, M_{Z}, M_{b}^{2}\right)\left(\frac{17}{18} M_{Z}^{2}-\frac{5}{18} \frac{M_{Z}^{4}}{M_{b}^{2}}+\frac{4}{9} M_{W}^{2}+\frac{2}{9} M_{W}^{2} \frac{M_{Z}^{2}}{M_{b}^{2}}-\frac{8}{9} \frac{M_{W}^{4}}{M_{Z}^{2}}-\frac{4}{9} \frac{M_{W}^{4}}{M_{b}^{2}}\right) \\
& \left.\sum_{f_{s}} A_{0}\left(m_{f}\right)\left(-4 \frac{m_{f}^{2}}{M_{H}^{2}}\right)\right) \\
& \delta M_{t}=M_{t} \mathcal{C}\left(-\frac{16}{9} s_{W}^{2} M_{W}^{2}+2 \frac{M_{Z}^{4}}{M_{H}^{2}}+4 \frac{M_{W}^{4}}{M_{H}^{2}}-\frac{1}{18} M_{Z}^{2}-\frac{29}{9} M_{W}^{2}+\frac{16}{9} \frac{M_{W}^{4}}{M_{Z}^{2}}\right. \\
& +A_{0}\left(M_{H}\right) \\
& +A_{0}\left(M_{W}\right)\left(\frac{1}{2}+6 \frac{M_{W}^{2}}{M_{H}^{2}}-\frac{1}{2} \frac{M_{b}^{2}}{M_{t}^{2}}-\frac{M_{W}^{2}}{M_{t}^{2}}\right) \\
& +A_{0}\left(M_{Z}\right)\left(3 \frac{M_{Z}^{2}}{M_{H}^{2}}-\frac{17}{18} \frac{M_{Z}^{2}}{M_{t}^{2}}+\frac{20}{9} \frac{M_{W}^{2}}{M_{t}^{2}}-\frac{16}{9} \frac{M_{W}^{2}}{M_{Z}^{2}} \frac{M_{W}^{2}}{M_{t}^{2}}\right) \\
& +A_{0}\left(M_{t}\right) 4 s_{W}^{2}\left(\frac{4}{3} \frac{M_{W}^{2}}{M_{t}^{2}}\right) \\
& +A_{0}\left(M_{t}\right)\left(1+\frac{17}{18} \frac{M_{Z}^{2}}{M_{t}^{2}}-\frac{20}{9} \frac{M_{W}^{2}}{M_{t}^{2}}+\frac{16}{9} \frac{M_{W}^{2}}{M_{Z}^{2}} \frac{M_{W}^{2}}{M_{t}^{2}}\right) \\
& +A_{0}\left(M_{b}\right)\left(\frac{1}{2}+\frac{1}{2} \frac{M_{b}^{2}}{M_{t}^{2}}+\frac{M_{W}^{2}}{M_{t}^{2}}\right) \\
& +B_{0}\left(M_{t}, M_{H}, M_{t}^{2}\right)\left(2 M_{t}^{2}-\frac{1}{2} M_{H}^{2}\right) \\
& +B_{0}\left(M_{t}, M_{Z}, M_{t}^{2}\right)\left(-\frac{7}{18} M_{Z}^{2}-\frac{17}{18} \frac{M_{Z}^{4}}{M_{t}^{2}}+\frac{40}{9} M_{W}^{2}+\frac{20}{9} M_{W}^{2} \frac{M_{Z}^{2}}{M_{t}^{2}}-\frac{32}{9} \frac{M_{W}^{4}}{M_{Z}^{2}}-\frac{16}{9} \frac{M_{W}^{4}}{M_{t}^{2}}\right) \\
& +B_{0}\left(M_{b}, M_{W}, M_{t}^{2}\right)\left(\frac{1}{2} \frac{M_{b}^{4}}{M_{t}^{2}}-M_{b}^{2}+\frac{1}{2} M_{t}^{2}+\frac{1}{2} \frac{M_{W}^{2}}{M_{t}^{2}} M_{b}^{2}+\frac{1}{2} M_{W}^{2}-\frac{M_{W}^{4}}{M_{t}^{2}}\right) \\
& \left.\sum_{f_{s}} A_{0}\left(m_{f}\right)\left(-4 \frac{m_{f}^{2}}{M_{H}^{2}}\right)\right)
\end{aligned}
$$


The on-loop corrections give the dominant contribution in the matching relations. Two-loop results may be found in Ref. [49] and in Refs. quoted in Sect. 3.

\section{References}

[1] G. Aad et al. [ATLAS Collaboration], Phys. Lett. B 716 (2012) 1; Science 338 (2012) 1576.

[2] S. Chatrchyan et al. [CMS Collaboration], Phys. Lett. B 716 (2012) 30; Science 338 (2012) 1569.

[3] T. Hambye, K. Riesselmann, Phys. Rev. D 55 (1997) 7255.

[4] M. Holthausen, K. S. Lim, M. Lindner, JHEP 1202 (2012) 037.

[5] F. Bezrukov, M.Yu. Kalmykov, B.A. Kniehl, M. Shaposhnikov, JHEP 1210 (2012) 140.

[6] G. Degrassi, S. Di Vita, J. Elias-Miro, J. R. Espinosa, G. F. Giudice, G. Isidori, A. Strumia, JHEP 1208 (2012) 098;

[7] S. Alekhin, A. Djouadi, S. Moch, Phys. Lett. B 716 (2012) 214.

[8] L. N. Mihaila, J. Salomon, M. Steinhauser, Phys. Rev. Lett. 108 (2012) 151602.

[9] K. G. Chetyrkin, M. F. Zoller, JHEP 1206 (2012) 033; JHEP 1304 (2013) 091.

[10] I. Masina, arXiv:1209.0393 [hep-ph].

[11] A. V. Bednyakov, A. F. Pikelner, V. N. Velizhanin, arXiv:1210.6873 [hep-ph]; arXiv:1212.6829 [hep-ph]; arXiv:1303.4364 [hep-ph].

[12] F. Jegerlehner,Quantum Field Theory and Statistical Mechanics, in 'Trends in Elementary Particle Theory", H. Rollnik, K. Dietz (eds.), Lecture Notes in Physics Vol 37, Springer, Berlin, 1975, p. 114-170; FUB-HEP-74-12.

[13] M. J. G. Veltman, Acta Phys. Polon. B 12 (1981) 437.

[14] F. Jegerlehner, Nucl. Phys. Proc. Suppl. 37B (1994) 129.

[15] M. J. G. Veltman, Nucl. Phys. B 7 (1968) 637.

[16] C. H. Llewellyn Smith, Phys. Lett. B 46 (1973) 233; J. S. Bell, Nucl. Phys. B 60 (1973) 427.

[17] J. M. Cornwall, D. N. Levin, G. Tiktopoulos, Phys. Rev. Lett. 30 (1973) 1268 [Erratum-ibid. 31 (1973) 572]; Phys. Rev. D 10 (1974) 1145 [Erratum-ibid. D 11 (1975) 972].

[18] F. Jegerlehner, Helv. Phys. Acta 51 (1978) 783;

[19] F. Jegerlehner, The *Ether-World* and elementary particles, in 'Theory of Elementary Particles', H. Dorn, D. Lüst, G. Weight (eds.), WILEY-VCH, Berlin, 1998, p. 386-392 hep-th/9803021].

[20] K. G. Wilson, Phys. Rev. B 4 (1971) 2174, ibid. 3184; K. G. Wilson, M. E. Fisher, Phys. Rev. Lett. 28 (1972) 204; K. G. Wilson, J. B. Kogut, Phys. Rept. 12 (1974) 75 . 
[21] F. Jegerlehner, M.Yu. Kalmykov, B.A. Kniehl, Phys.Lett. B 722 (2013) 123.

[22] H. Aoki, S. Iso, Phys. Rev. D 86 (2012) 013001.

[23] M. S. Al-sarhi, I. Jack, D. R. T. Jones, Z. Phys. C 55 (1992) 283.

[24] Y. Hamada, H. Kawai, K.Y. Oda, Phys. Rev. D 87 (2013) 053009

[25] A. Pais, G. E. Uhlenbeck, Phys. Rev. 79 (1950) 145.

[26] W. Pauli, F. Villars, Rev. Mod. Phys. 21 (1949) 434.

[27] F. Jegerlehner, Phys. Rev. D 16 (1977) 397.

[28] K. Symanzik, Commun. Math. Phys. 45 (1975) 79.

[29] J. Zinn-Justin, in Cargèse Lectures in Physics, 1973, Ed. E. Brèzin (unpublished); E. Brèzin, J. C. Le Guillou, J. Zinn-Justin, in Phase Transitions and Critical Phenomena, edited by C. Domb and M. S. Green (Academic, New York, 1976) Vol. 6, 125-247.

[30] C. G. Callan, Jr., Phys. Rev. D 2 (1970) 1541.

[31] K. Symanzik, Commun. Math. Phys. 18 (1970) 227.

[32] G. 't Hooft, M. Veltman, Nucl. Phys. B 44 (1972) 189.

[33] G. 't Hooft, Nucl. Phys. B 61 (1973) 455.

[34] J. Fleischer, F. Jegerlehner, Phys. Rev. D 23 (1981) 2001.

[35] A. Sirlin, Phys. Rev. D 22 (1980) 971;

A. Sirlin, R. Zucchini, Nucl. Phys. B 266 (1986) 389.

[36] N. Gray, D.J. Broadhurst, W. Grafe, K. Schilcher, Z. Phys. C 48 (1990) 673; J. Fleischer, F. Jegerlehner, O. V. Tarasov, O. L. Veretin, Nucl. Phys. B 539 (1999) 671 [Erratum-ibid. B 571 (2000) 511].

[37] K.G. Chetyrkin, M. Steinhauser, Phys. Rev. Lett. 83 (1999) 4001; Nucl. Phys. B 573 (2000) 617.

[38] K. Melnikov, T. v. Ritbergen, Phys. Lett. B 482 (2000) 99.

[39] A.L. Kataev, V.T. Kim, Phys. Part. Nucl. 41 (2010) 946.

[40] R. Hempfling, B.A. Kniehl, Phys. Rev. D 51 (1995) 1386.

[41] F. Jegerlehner, M.Yu. Kalmykov, Nucl. Phys. B 676 (2004) 365; Acta Phys. Polon. B 34 (2003) 5335; Nucl. Instrum. Meth. A 534 (2004) 299.

[42] S.P. Martin, Phys. Rev. D 72 (2005) 096008.

[43] D. Eiras, M. Steinhauser, J. High Energy Phys. 0602 (2006) 010.

[44] M. Faisst, J.H. Kühn, O. Veretin, Phys. Lett. B 589 (2004) 35.

[45] T. Appelquist, J. Carazzone, Phys. Rev. D 11 (1975) 2856.

[46] J.C. Taylor, Gauge Theories of Weak Interactions, Cambridge Monographs on Mathematical Physics, 1976, pp. 124-127;

E. Kraus, Annals Phys. 262 (1998) 155. 
[47] J. Beringer et al. [Particle Data Group Collaboration], Phys. Rev. D 86 (2012) 010001.

[48] D.R.T. Jones, Nucl. Phys. B87 (1975) 127; Phys. Rev. D25 (1982) 581;

M.E. Machacek, M.T. Vaughn, Nucl.Phys. B222 (1983) 83.

[49] F. Jegerlehner, M.Yu. Kalmykov, O. Veretin, Nucl. Phys. B 641 (2002) 285; Nucl. Phys. B (Proc. Suppl.) 116 (2003) 382; Nucl. Phys. B 658 (2003) 49.

[50] M.J.G. Veltman, Acta Phys. Polon. B 12 (1981) 437.

[51] A. H. Guth, Phys. Rev. D 23 (1981) 347.

[52] A. A. Starobinsky, Phys. Lett. B 91 (1980) 99.

[53] A. D. Linde, Phys. Lett. B 108 (1982) 389, Phys. Lett. B 129 (1983) 177.

[54] A. Albrecht, P. J. Steinhardt, Phys. Rev. Lett. 48 (1982) 1220.

[55] V. F. Mukhanov, G. V. Chibisov, JETP Lett. 33 (1981) 532 [Pisma Zh. Eksp. Teor. Fiz. 33 (1981) 549]; V. F. Mukhanov, JETP Lett. 41 (1985) 493 [Pisma Zh. Eksp. Teor. Fiz. 41 (1985) 402]; V. F. Mukhanov, H. A. Feldman, R. H. Brandenberger, Phys. Rept. 215 (1992) 203.

[56] P. A. R. Ade et al. [Planck Collaboration], arXiv:1303.5062 [astro-ph.CO]; arXiv:1303.5082 [astro-ph.CO]; arXiv:1303.5084 [astro-ph.CO].

[57] W. Buchmüller, Z. Fodor, A. Hebecker, Nucl. Phys. B 447 (1995) 317.

[58] V. A. Rubakov, M. E. Shaposhnikov, Usp. Fiz. Nauk 166 (1996) 493 [Phys. Usp. 39 (1996) 461] hep-ph/9603208].

[59] N. Straumann, Eur. J. Phys. 20 (1999) 419. 